\title{
Modelling of turbulence modulation in particle- or droplet-laden flows
}

\author{
Journal Article
}

Author(s):

Meyer, Daniel W.

Publication date:

2012-09

Permanent link:

https://doi.org/10.3929/ethz-b-000055596

Rights / license:

In Copyright - Non-Commercial Use Permitted

Originally published in:

Journal of Fluid Mechanics 706, https://doi.org/10.1017/jfm.2012.251 


\title{
Modelling of turbulence modulation in particle- or droplet-laden flows
}

\author{
Daniel W. Meyer $\dagger$ \\ Institute of Fluid Dynamics, ETH Zurich, Sonneggstrasse 3, CH-8092 Zürich, Switzerland
}

(Received 2 February 2012; revised 3 May 2012; accepted 23 May 2012;

first published online 12 July 2012)

Addition of particles or droplets to turbulent liquid flows or addition of droplets to turbulent gas flows can lead to modulation of turbulence characteristics. Corresponding observations have been reported for very small particle or droplet volume loadings $\Phi_{v}$ and therefore may be important when simulating such flows. In this work, a modelling framework that accounts for preferential concentration and reproduces isotropic and anisotropic turbulence attenuation effects is presented. The framework is outlined for both Reynolds-averaged Navier-Stokes (RANS) and joint probability density function (p.d.f.) methods. Validations are performed involving a range of particle and flow-field parameters and are based on the direct numerical simulation (DNS) study of Boivin, Simonin \& Squires (J. Fluid Mech., vol. 375, 1998, pp. 235-263) dealing with heavy particles suspended in homogeneous isotropic turbulence (Stokes number $S t=O(1-10)$, particle/fluid density ratio $\rho_{p} / \rho=2000, \Phi_{v}=O\left(10^{-4}\right)$ ) and the experimental investigation of Poelma, Westerweel \& Ooms (J. Fluid Mech., vol. 589, 2007, pp. 315-351) involving light particles $\left(S t=O(0.1), \rho_{p} / \rho \gtrsim 1, \Phi_{v}=O\left(10^{-3}\right)\right)$ settling in grid turbulence. The development in this work is restricted to volume loadings where particle or droplet collisions are negligible.

Key words: multiphase flow, turbulence modelling

\section{Introduction}

Turbulent dispersed multiphase flows that involve particles or droplets dispersed in turbulent carrier flows are present in many processes in nature and industry. For example, droplet-turbulence interaction influences rain and cloud formation (Shaw 2003). Spray combustion or aerosol transport, for example, are relevant industrial and environmental applications (Fernando \& Choi 2007). Based on the high relevance of dispersed multiphase flows in industrial and environmental processes, various reviews have been compiled over the past few years dealing with the simulation of particleor droplet-laden flows (Loth 2000; Eaton 2006, 2009; Balachandar \& Eaton 2010). Henceforth, the term particle is used generically to cover both particles and droplets.

The fluid and particle phases are characterized by a small number of parameters. The turbulence of the continuous fluid phase is described by the fluid density $\rho$ and viscosity $v$, the integral length scale $L$, the Kolmogorov length and time scales $\eta$ and $\tau_{\eta}$, respectively, the turbulent kinetic energy $k$, and its dissipation rate $\varepsilon$. The particle phase is characterized by the particle diameter $d$, the particle density $\rho_{p}$, 
and the volume loading $\Phi_{v}$. The latter is equal to the ratio of volume fractions occupied by the particle and fluid phases. In this work, we focus on a monodisperse particle phase where particles of one size are present, but extensions for polydisperse particles are discussed as well. Different interaction regimes between the two phases can be distinguished based on $\Phi_{v}$ and were summarized by Poelma, Westerweel \& Ooms (2007, table 1). For $\Phi_{v}<10^{-6}$, the dispersed phase is influenced by the fluid-phase turbulence but not vice versa. In this regime, the interaction between the fluid and particle phases is referred to as one-way coupling. At intermediate loadings, $10^{-5}<\Phi_{v}<10^{-2}$, both phases interact, which is called two-way coupling. If $\Phi_{v}$ is increased above $10^{-2}$, the particles not only have an effect on the fluid phase but also collide (four-way coupling). In this work, we focus on the two-way coupling regime.

Interphase processes of different form and complexity can be characterized by a set of parameters. The Stokes relaxation time, defined as

$$
\tau_{p} \equiv \frac{2}{9} \frac{\rho_{p}}{\rho} \frac{1}{v}\left(\frac{d}{2}\right)^{2}
$$

(see Squires \& Eaton 1991a, equation (3.14)), quantifies the drag-driven particle response time to velocity variations in the fluid phase. Particles with small $\tau_{p}$ adjust quickly to velocity-field changes in the carrier flow. Based on the parameters listed in the previous paragraph, a set of non-dimensional parameters can be formulated: the length scale and density ratios $d / \eta$ and $\rho_{p} / \rho$, respectively, the mass loading $\Phi_{m} \equiv\left(\rho_{p} / \rho\right) \Phi_{v}$, the Stokes number $S t \equiv \tau_{p} / \tau_{\eta}$, and the particle Reynolds number $R e_{p} \equiv|\boldsymbol{u}-\boldsymbol{v}| d / \nu$. Here, $|\boldsymbol{u}-\boldsymbol{v}|$ is the magnitude of the relative velocity between the particle velocity $\boldsymbol{v}$ and the surrounding carrier fluid flow $\boldsymbol{u}$.

For very small $S t$ and in the absence of external forces like gravity, particles behave like passive tracers and are convected with the carrier flow, i.e. $R e_{p} \approx 0$. For increased $S t$ or when external forces are present, particles no longer move synchronously with the carrier flow; therefore $R e_{p}>0$, and the relative motion between the fluid and particle phases leads, at sufficiently high loadings $\left(\Phi_{v}>10^{-5}\right)$, to modulations of the carrier-phase turbulence (Eaton 2006; Balachandar \& Eaton 2010). Boivin, Simonin \& Squires (1998, figure 3) have demonstrated by means of direct numerical simulation (DNS) that in the absence of external forces particles with Stokes numbers in the range from 1 to 10 can reduce the turbulence intensity, i.e. $k$, by more than $50 \%$ for $\Phi_{v}=O\left(10^{-4}\right)$ and $\Phi_{m}=1$. This illustrates that even for very small volume loadings, two-way coupling effects can be significant. More recently, Poelma et al. (2007) have demonstrated experimentally that particles with $S t=O(0.1)$ settling under gravity may induce an anisotropic dissipation tensor $\varepsilon_{i j}$ in the evolution equation of the fluid-phase Reynolds stress tensor $\left\langle u_{i} u_{j}\right\rangle$. In the experiments of Geiss et al. (2004) and the DNS of Elghobashi \& Truesdell (1992) and Elghobashi (1993) similar effects were documented.

Moreover, at moderate Stokes numbers, i.e. St $\approx 1$, and with $\rho_{p} / \rho>1$, particles accumulate away from flow regions with high vorticity, which is referred to as preferential concentration (Balachandar \& Eaton 2010, § 4.1). A number of DNS studies dealing with preferential concentration in homogeneous turbulence have been conducted, for example by Squires \& Eaton (1990, 1991b), He et al. (2005), and Jung, Yeo \& Lee (2008). Also by means of DNS, Sundaram \& Collins (1997) have demonstrated that preferential concentration is important in flows where particle collisions matter. A review of preferential concentration effects in various flows was compiled by Eaton \& Fessler (1994). If $\tau_{p} \gg \tau_{\eta}$ and $S t \gg 1$, the turbulent fluid-phase 
motions have virtually no influence on the particle motions. However, if the particles are sufficiently large and $R e_{p}$ exceeds the critical Reynolds number of around 210 (Bagchi \& Balachandar 2004, p. 121), vortex shedding occurs in the particle wakes, which leads to turbulence augmentation (Balachandar \& Eaton 2010, §6.2). More specifically, Eaton (2006, p. 12-87) and Balachandar \& Eaton (2010, p. 125) conclude that particles with diameters $>0.1 L$ lead to turbulence augmentation whereas smaller ones lead to turbulence attenuation. In this work, we focus on quite small particle Reynolds numbers $R e_{p} \approx 0.3-30$ and therefore ignore turbulence augmentation due to vortex shedding.

Different simulation methods are applied for the simulation of turbulent particleladen flows. Loth (2000) has reviewed contributions that focus on the one-way coupling regime and has included Reynolds-averaged Navier-Stokes (RANS) methods, large-eddy simulation (LES), and DNS. For the treatment of the particle phase, Loth has distinguished between Eulerian (based on conservation laws) and particle-based Lagrangian methods. The Lagrangian methods were further decomposed into pointvolume and resolved-volume particle methods (Loth 2000, figure 4). Point-volume particle methods do not resolve the particle geometry and neglect particle volume effects on the continuous fluid phase. These methods are computationally efficient, but applicable for $d<\eta$ only.

For example, Minier, Peirano \& Chibbaro (2004) have proposed an Eulerian/Lagrangian point-volume simulation method, where an Eulerian grid-based technique was applied to solve the RANS equations for the fluid phase and a Lagrangian probability density function (p.d.f.) method was used for the particle phase. Their method is applicable for heavy particles with $\rho_{p} \gg \rho$ and accounts for two-way coupling effects on the basis of the $k$-equation. Accordingly, anisotropic turbulence modulation effects as documented by Geiss et al. (2004) and Poelma et al. (2007) cannot be accounted for. Moreover, preferential concentration effects are not addressed in the framework of Minier et al. (2004). However, model predictions were found to be in good agreement with an experiment involving a particle-laden bluff-body flow.

More recently, Balachandar \& Eaton $(2010, \S 3)$ have reviewed LES and DNS simulation approaches. There, and also in other contributions focused on DNS (e.g. Boivin et al. (1998) or Eaton (2006)), the point-volume particle method is referred to as the point-force approximation or point-force coupling scheme. Shortcomings of the point-force approximation were discussed by Eaton (2006, top of p. 12-91). Balachandar \& Eaton (2010) and also Eaton (2006, § 12.6.5) have discussed turbulence modulation effects and concluded that improved models are needed for simulations in the two-way coupling regime.

In the present work, a refined Eulerian/Lagrangian point-volume simulation framework similar to the one proposed by Minier \& Peirano (2001) is outlined. We take the contributions of Boivin et al. (1998) and Poelma et al. (2007) as a reference and develop models that can reproduce the reported turbulence modulation effects for a range of particle and loading parameters. As outlined previously, the studies of Boivin et al. (1998) and Poelma et al. (2007) focus on well-documented turbulence modulation effects and deal with different canonical flows. Both studies provide details about the selected flow and particle parameters and quantify turbulence modulation effects based on quantities that are available in the RANS context. Moreover, in both studies, $d<\eta$ and in the DNS study of Boivin et al. (1998), the point-force approximation is applied.

The main contributions of the present work are as follows. A stochastic Lagrangian model for the particle phase is outlined that accounts for preferential concentration, 
anisotropy in the fluid-phase Reynolds stress tensor, and enforces consistency between the seen and fluid-phase statistics. Based on the experimental work of Poelma et al. (2007), a refined model for the dissipation tensor in the fluid-phase Reynolds stress transport equation is proposed. It accounts for anisotropic dissipation effects and formulations for both RANS and p.d.f. methods are presented. Moreover, a new nondimensional group is introduced that relates dissipation anisotropy to the underlying particle- and fluid-phase characteristics. In $\S 2$, the governing equations for the fluid and particle phases are presented and the model formulations are outlined both in the RANS ( $\S 2.2$ and 2.3) and p.d.f. context (§2.4). Section 3 deals with the validation of the modelling framework based on the DNS data of Boivin et al. (1998) and the experimental study of Poelma et al. (2007). Finally, conclusions are drawn in $\S 4$.

\section{Formulation}

In several previous contributions dealing with dispersed two-phase flow (Squires \& Eaton 1990; Elghobashi 1993; Boivin et al. 1998), the point-force approximation was applied. Here, the two-way coupling forces at the particle/fluid interface are represented in the Navier-Stokes equation by Dirac delta functions, i.e.

$$
\frac{\mathrm{D} \hat{\boldsymbol{u}}}{\mathrm{D} t} \equiv \frac{\partial \hat{\boldsymbol{u}}}{\partial t}+(\hat{\boldsymbol{u}} \cdot \nabla) \hat{\boldsymbol{u}}=-\frac{1}{\rho} \nabla \hat{p}+v \nabla^{2} \hat{\boldsymbol{u}}+\frac{1}{\rho} \sum_{n=1}^{N}\left[\boldsymbol{F}^{n} \boldsymbol{\delta}\left(\boldsymbol{x}-\boldsymbol{x}^{n}\right)\right],
$$

where $\hat{p}$ is the pressure, $\boldsymbol{F}^{n}$ and $\boldsymbol{x}^{n}$ are the drag force experienced by particle $n$ and the position of particle $n=1 \ldots N$, respectively, with $N$ dispersed particles in total. $\boldsymbol{\delta}\left(\boldsymbol{x}-\boldsymbol{x}^{n}\right) \equiv \prod_{i=1}^{3} \delta\left(x_{i}-x_{i}^{n}\right)$ is a vectorial Dirac delta function with $\boldsymbol{\delta}\left(\boldsymbol{x}-\boldsymbol{x}^{n}\right)=0$ for $\boldsymbol{x} \neq \boldsymbol{x}^{n}$ and

$$
\int_{-\infty}^{\infty} \boldsymbol{\delta}\left(\boldsymbol{x}-\boldsymbol{x}^{n}\right) \mathrm{d} \boldsymbol{x} \equiv \int_{-\infty}^{\infty} \int_{-\infty}^{\infty} \int_{-\infty}^{\infty} \boldsymbol{\delta}\left(\boldsymbol{x}-\boldsymbol{x}^{n}\right) \mathrm{d} x_{1} \mathrm{~d} x_{2} \mathrm{~d} x_{3}=1 .
$$

Gravity effects may be included in (2.1) but can be lumped into a modified pressure $\hat{p}_{g}$ by means of a suitable potential, e.g. $\hat{p}_{g} \equiv \hat{p}-\rho g x_{1}$. Here, $g$ is the gravity constant. The position $\boldsymbol{x}^{n}(t)$ of particle $n$ with velocity $\boldsymbol{v}^{n}(t)$ is given by

$$
\frac{\mathrm{d} \boldsymbol{x}^{n}}{\mathrm{~d} t}=\boldsymbol{v}^{n}
$$

and

$$
m_{p} \frac{\mathrm{d} \boldsymbol{v}^{n}}{\mathrm{~d} t}=-\underbrace{m_{p} \frac{c}{\tau_{p}}\left[\boldsymbol{v}^{n}-\boldsymbol{u}\left(\boldsymbol{x}^{n}, t\right)\right]}_{\equiv \boldsymbol{F}^{n}}+m_{p} \frac{\rho}{\rho_{p}} \frac{\mathrm{D} \boldsymbol{u}}{\mathrm{D} t}+m_{p}\left(1-\frac{\rho}{\rho_{p}}\right) \boldsymbol{g}
$$

(Balachandar \& Eaton 2010, p. 123). In (2.4), $m_{p}$ is the mass of a single particle, $\boldsymbol{g}$ is the gravitational acceleration vector, and $\boldsymbol{F}^{n}$ is the Stokes drag force. In the definition of $\boldsymbol{F}^{n}, \boldsymbol{u}\left(\boldsymbol{x}^{n}, t\right)$ is the velocity of the fluid that is surrounding particle $n$ but is not disturbed by particle $n ; \boldsymbol{u}\left(\boldsymbol{x}^{n}, t\right)$ at particle location $\boldsymbol{x}^{n}$ is also referred to as seen fluid-phase velocity. To account for nonlinear drag effects at elevated particle Reynolds numbers $R e_{p}$, the Schiller-Naumann drag correction $c \equiv 1+0.15 R e_{p}^{0.687}$ is applied with

$$
\operatorname{Re}_{p} \equiv \frac{\left|\boldsymbol{v}^{n}-\boldsymbol{u}\left(\boldsymbol{x}^{n}, t\right)\right| d}{v}
$$


The second term on the right-hand side of (2.4) represents pressure and viscous forces exerted by the fluid phase on particle $n$. The last term in (2.4) represents gravity and buoyancy effects.

For small particles with $d \ll \eta$, the fluid-phase velocity in the undisturbed particle neighbourhood, i.e. $\boldsymbol{u}\left(\boldsymbol{x}^{n}, t\right)$, is uniform to a good approximation but $\neq \hat{\boldsymbol{u}}\left[\boldsymbol{x}^{n}(t), t\right]$ from (2.1). To determine $\boldsymbol{u}(\boldsymbol{x}, t)$ from the velocity field $\hat{\boldsymbol{u}}(\boldsymbol{x}, t)$, a low-pass filter of width $\Delta$ in the range $d \ll \Delta<\eta$ is applied. The filtering removes the localized flow-field disturbances induced by the particles and leads to a suitable approximation for $\boldsymbol{u}(\boldsymbol{x}, t)$. To this end, (2.1) is multiplied by the box filter kernel defined as

$$
G(\boldsymbol{r})=\frac{1}{\Delta^{3}} \prod_{i=1}^{3} H\left(\frac{1}{2} \Delta-\left|r_{i}\right|\right)
$$

with $H(r)$ being the Heaviside step function. Subsequently, (2.1) is integrated with respect to the displacement vector $\boldsymbol{r}$. Unlike in LES, where typically the filter width $\Delta \gg \eta$, here the filtering operation has little effect on the structure of (2.1). For the time-derivative term, for example, we obtain

$$
\int_{-\infty}^{\infty} G(\boldsymbol{r}) \frac{\partial \hat{\boldsymbol{u}}(\boldsymbol{x}-\boldsymbol{r}, t)}{\partial t} \mathrm{~d} \boldsymbol{r}=\frac{\partial}{\partial t} \underbrace{\int_{-\infty}^{\infty} G(\boldsymbol{r}) \hat{\boldsymbol{u}}(\boldsymbol{x}-\boldsymbol{r}, t) \mathrm{d} \boldsymbol{r}}_{\equiv \boldsymbol{u}(\boldsymbol{x}, t)}=\frac{\partial \boldsymbol{u}(\boldsymbol{x}, t)}{\partial t} .
$$

The last term reduces to

$$
\begin{aligned}
& \frac{1}{\rho} \sum_{n=1}^{N}\left\{\boldsymbol{F}^{n}(t) \int_{-\infty}^{\infty} G(\boldsymbol{r}) \boldsymbol{\delta}\left[\boldsymbol{x}-\boldsymbol{r}-\boldsymbol{x}^{n}(t)\right] \mathrm{d} \boldsymbol{r}\right\} \\
& =\frac{1}{\rho} \sum_{n=1}^{N}\left\{\boldsymbol{F}^{n}(t) G\left[\boldsymbol{x}-\boldsymbol{x}^{n}(t)\right] \int_{-\infty}^{\infty} \boldsymbol{\delta}\left[\boldsymbol{x}-\boldsymbol{r}-\boldsymbol{x}^{n}(t)\right] \mathrm{d} \boldsymbol{r}\right\} \\
& =\frac{1}{\Delta^{3} \rho} \sum_{n=1}^{N} \boldsymbol{F}^{n}(t) \underbrace{\prod_{i=1}^{1} H\left[\frac{1}{2} \Delta-\left|x_{i}-x_{i}^{n}(t)\right|\right]}_{=} \\
& \quad=\frac{m_{p}}{\Delta^{3} \rho} \boldsymbol{f}(\boldsymbol{x}, t) n_{p}(\boldsymbol{x}, t)
\end{aligned}
$$

with $\boldsymbol{f}(\boldsymbol{x}, t)$ being the algebraic mean of the Stokes drag forces $\boldsymbol{F}^{n}(t) / m_{p}$ of all $n_{p}(\boldsymbol{x}, t)$ particles inside the filter volume located at $\boldsymbol{x}$. Finally, with the mass loading

$$
\Phi_{m}(\boldsymbol{x}, t) \equiv \frac{m_{p} n_{p}(\boldsymbol{x}, t)}{\Delta^{3} \rho},
$$

the filtered Navier-Stokes equation reduces to

$$
\frac{\partial \boldsymbol{u}}{\partial t}+(\boldsymbol{u} \cdot \nabla) \boldsymbol{u}=-\frac{1}{\rho} \nabla p+v \nabla^{2} \boldsymbol{u}+\Phi_{m} \boldsymbol{f},
$$

where $p(\boldsymbol{x}, t)$ is the filtered pressure. Equation (2.10) is equivalent to equations applied, for example, by Squires \& Eaton (1990, p. 1193) and Rogers \& Eaton (1991, p. 935), and discussed in the review articles by Eaton $(2006, \S 12.6 .3)$ and Balachandar \& Eaton (2010, p. 127). In the DNS studies of Boivin et al. (1998, § 2.5) and 
Elghobashi (1993, p. 1791), the effect of the point forces in the two-way coupling term in (2.1) is distributed to neighbouring grid nodes of grid cells with size $\lesssim \eta$. This treatment has the same effect on $\hat{\boldsymbol{u}}(\boldsymbol{x}, t)$ as the previously outlined filtering.

\subsection{Reynolds-averaged equations}

Next, RANS equations are discussed for the computationally efficient solution of particle-laden flows. Ensemble averaging the Navier-Stokes equation (2.10) leads for velocity component $u_{i}$ (Pope 2000, p. 85) to

$$
\frac{\partial\left\langle u_{i}\right\rangle}{\partial t}+\left\langle u_{j}\right\rangle \frac{\partial\left\langle u_{i}\right\rangle}{\partial x_{j}}=-\frac{1}{\rho} \frac{\partial\langle p\rangle}{\partial x_{i}}+v \frac{\partial^{2}\left\langle u_{i}\right\rangle}{\partial x_{j} \partial x_{j}}-\frac{\partial\left\langle u_{i}^{\prime} u_{j}^{\prime}\right\rangle}{\partial x_{j}}+\left\langle\Phi_{m} f_{i}\right\rangle .
$$

In (2.11), the Reynolds decomposition with the fluctuating velocity defined as $\boldsymbol{u}^{\prime} \equiv \boldsymbol{u}-\langle\boldsymbol{u}\rangle$ was used. Equation (2.11) has a similar structure to the Navier-Stokes equation (2.10), but the ensemble-averaged quantities show much less spatial variability (Pope 2000, pp. 556-557) and, therefore, much coarser computational grids can be used for its numerical solution. However, the Reynolds stress tensor $\left\langle u_{i}^{\prime} u_{j}^{\prime}\right\rangle$ and the two-way coupling term, i.e. $\left\langle\Phi_{m} f_{i}\right\rangle$, require closure relations. Corresponding expressions are discussed in the following three sections.

A transport equation for $\left\langle u_{i}^{\prime} u_{j}^{\prime}\right\rangle$ is derived with the procedure outlined by Fox (2003, $\S 2.2 .3)$ that is based on the relation

$$
\frac{\partial u_{i}^{\prime} u_{j}^{\prime}}{\partial y}=u_{j}^{\prime} \frac{\partial u_{i}^{\prime}}{\partial y}+u_{i}^{\prime} \frac{\partial u_{j}^{\prime}}{\partial y},
$$

where $y$ is a generic independent variable representing $t$ or an arbitrary spatial coordinate $x_{k}$. Subtracting RANS equation (2.11) from Navier-Stokes equation (2.10) for component $u_{i}$ leads to an equation for $u_{i}^{\prime}$. Multiplication of this equation by $u_{j}^{\prime}$ and addition of a corresponding equation for $u_{j}^{\prime}$ multiplied by $u_{i}^{\prime}$ leads after ensemble averaging to the Reynolds stress transport equation

$$
\begin{aligned}
\frac{\partial\left\langle u_{i}^{\prime} u_{j}^{\prime}\right\rangle}{\partial t}+\left\langle u_{k}\right\rangle \frac{\partial\left\langle u_{i}^{\prime} u_{j}^{\prime}\right\rangle}{\partial x_{k}}= & -\frac{\partial\left\langle u_{i}^{\prime} u_{j}^{\prime} u_{k}^{\prime}\right\rangle}{\partial x_{k}}+\mathscr{P}_{i j}+\Pi_{i j}+v \frac{\partial^{2}\left\langle u_{i}^{\prime} u_{j}^{\prime}\right\rangle}{\partial x_{k} \partial x_{k}}-\varepsilon_{i j} \\
& +\left\langle\Phi_{m} u_{j}^{\prime} f_{i}\right\rangle+\left\langle\Phi_{m} u_{i}^{\prime} f_{j}\right\rangle
\end{aligned}
$$

with the production term

$$
\mathscr{P}_{i j} \equiv\left\langle u_{i}^{\prime} u_{k}^{\prime}\right\rangle \frac{\partial\left\langle u_{j}\right\rangle}{\partial x_{k}}+\left\langle u_{j}^{\prime} u_{k}^{\prime}\right\rangle \frac{\partial\left\langle u_{i}\right\rangle}{\partial x_{k}},
$$

the velocity-pressure-gradient term

$$
\Pi_{i j} \equiv-\frac{1}{\rho}\left\langle u_{i}^{\prime} \frac{\partial p^{\prime}}{\partial x_{j}}+u_{j}^{\prime} \frac{\partial p^{\prime}}{\partial x_{i}}\right\rangle,
$$

and the dissipation tensor

$$
\varepsilon_{i j} \equiv 2 v\left\langle\frac{\partial u_{i}^{\prime}}{\partial x_{k}} \frac{\partial u_{j}^{\prime}}{\partial x_{k}}\right\rangle
$$

(except for the two-phase flow terms see Fox (2003, pp. 49-50)). The last two terms appear in unclosed form and for example $\varepsilon_{i j}$ is often approximated by the isotropic model $\varepsilon_{i j}=(2 / 3) \varepsilon \delta_{i j}$ away from walls (Pope 2000, p. 388). Here, $\delta_{i j}$ is the Kronecker delta. 
For the turbulent kinetic energy $k \equiv(1 / 2)\left\langle u_{i}^{\prime} u_{i}^{\prime}\right\rangle,(2.13)$ reduces to

$$
\begin{aligned}
\frac{\partial k}{\partial t}+\left\langle u_{j}\right\rangle \frac{\partial k}{\partial x_{j}}= & -\frac{1}{2} \frac{\partial\left\langle u_{i}^{\prime} u_{i}^{\prime} u_{j}^{\prime}\right\rangle}{\partial x_{j}}-\frac{1}{\rho} \frac{\partial\left\langle u_{j}^{\prime} p^{\prime}\right\rangle}{\partial x_{j}}+v \frac{\partial^{2}\left\langle u_{i}^{\prime} u_{j}^{\prime}\right\rangle}{\partial x_{i} \partial x_{j}}+\mathscr{P} \\
& +v \frac{\partial^{2} k}{\partial x_{j} \partial x_{j}}-\varepsilon+\left\langle\Phi_{m} u_{i}^{\prime} f_{i}\right\rangle
\end{aligned}
$$

with the kinetic energy production and dissipation rate

$$
\mathscr{P} \equiv\left\langle u_{i}^{\prime} u_{j}^{\prime}\right\rangle \frac{\partial\left\langle u_{i}\right\rangle}{\partial x_{j}} \quad \text { and } \varepsilon \equiv \underbrace{v\left\langle\frac{\partial u_{i}^{\prime}}{\partial x_{j}} \frac{\partial u_{i}^{\prime}}{\partial x_{j}}\right\rangle}_{\equiv \tilde{\varepsilon}}+v \frac{\partial^{2}\left\langle u_{i}^{\prime} u_{j}^{\prime}\right\rangle}{\partial x_{i} \partial x_{j}},
$$

respectively (Fox (2003, p. 51); Pope (2000, pp. 132-133)); $\tilde{\varepsilon}$ is the pseudodissipation.

For forced homogeneous isotropic turbulence with $\langle\boldsymbol{v}\rangle=0$ and $\langle\boldsymbol{u}\rangle=0, \partial k / \partial t=0$ (or more precisely $\mathrm{d} k / \mathrm{d} t=0$ since $t$ remains the only independent variable), and all particles uniformly distributed, i.e. $\Phi_{m}$ being constant, (2.17) simplifies to

$$
\frac{\mathrm{d} k}{\mathrm{~d} t}=\mathscr{P}_{f}-\varepsilon+\mathscr{P}_{S t}=0 \quad \text { with } \mathscr{P}_{S t} \equiv \Phi_{m}\left\langle u_{i}^{\prime} f_{i}\right\rangle
$$

similar to Boivin et al. (1998, equation (3.1)). Here, $\mathscr{P}_{f}$ is an artificial forcing term that was added to the $k$-equation to keep $k$ constant over time. To implement $\mathscr{P}_{f}$, for example Boivin et al. (1998) have used in their spectral DNS the forcing scheme proposed by Eswaran \& Pope (1988), where kinetic energy is added by random excitation of the large-scale modes. $-\mathscr{P}_{S t}$ in (2.19) is referred to by Eaton (2006, p. 12-90) and Balachandar \& Eaton (2010, p. 127) as particle extra dissipation.

\subsection{Lagrangian particle-phase model}

In this section, a new model is outlined that mimics the dynamics of particles and enables the calculation of unclosed two-way coupling terms like $\left\langle\Phi_{m} f_{i}\right\rangle$ or $\left\langle\Phi_{m} u_{i}^{\prime} f_{i}\right\rangle$ based on an ensemble of particles. To this end, a new stochastic model for the seen fluid phase velocity $\boldsymbol{u}\left(\boldsymbol{x}^{n}\right)$ and the Lagrangian fluid-phase acceleration $\mathrm{D} \boldsymbol{u} / \mathrm{D} t$, both appearing in (2.4), is proposed. Stochastic processes for the Lagrangian fluid acceleration were developed in the context of p.d.f. methods by Pope $(1985, \S 4.6)$. However, since particles are not necessarily passively convected with the fluid flow, modified processes are needed. In the limit of small or large Stokes numbers, particles behave like fluid particles or heavy ballistic particles, respectively, both experiencing the same local velocity statistics characterized by $\langle\boldsymbol{u}\rangle$ and $\left\langle u_{i}^{\prime} u_{j}^{\prime}\right\rangle$. In the present work, it is assumed that the seen velocity is characterized by $\langle\boldsymbol{u}\rangle$ and $\left\langle u_{i}^{\prime} u_{j}^{\prime}\right\rangle$ from (2.11) and (2.13) irrespective of the Stokes number.

To make sure that the seen velocity statistics are consistent with the mean fluid velocity and Reynolds stress tensor at position $\boldsymbol{x}^{n}(t)$ the following numerical scheme is applied. The Reynolds stress tensor $\boldsymbol{U}$ with elements $\left\langle u_{i}^{\prime} u_{j}^{\prime}\right\rangle$ is a symmetric, diagonalizable matrix. Therefore, $\boldsymbol{V}^{\mathrm{T}} \boldsymbol{U} \boldsymbol{V}=\boldsymbol{W}$, where matrix $\boldsymbol{V}$ and the diagonal matrix $\boldsymbol{W}$ contain the eigenvectors and eigenvalues of tensor $\boldsymbol{U}$, respectively. At every spatial location or in every computational grid cell, the diagonalized Reynolds stress tensor $\boldsymbol{W}$ can be computed for example by a QL-decomposition-based method as documented by Press $(2001, \S 11)$. In the principal-axes coordinate system where $\boldsymbol{W}$ is defined, the cross-covariances $\left\langle w_{i}^{\prime} w_{j}^{\prime}\right\rangle=0 \forall i \neq j$. If we assume that the local velocity p.d.f. is joint 
Gaussian, the velocity components $w_{i}^{\prime}$ are statistically independent processes (Pope 2000, p. 63). Therefore, to model $\boldsymbol{u}\left(\boldsymbol{x}^{n}\right)$ and $\mathrm{D} \boldsymbol{u} / \mathrm{D} t$ we attribute to every particle three independent Ornstein-Uhlenbeck processes $\xi_{i}^{n}(t)$ with correlation time scales $T_{L}^{*}$ and standard normal stationary probability distribution, i.e.

$$
\mathrm{d} \xi_{i}^{n}=-\frac{\mathrm{d} t}{T_{L}^{*}} \xi_{i}^{n}+\sqrt{\frac{2}{T_{L}^{*}}} \mathrm{~d} W_{i}(t),
$$

where $\mathrm{d} t$ is the time step size and $\mathrm{d} W_{i}(t)$ is a Wiener process increment that is statistically independent for every component $i$ and particle $n$ (Pope 2000, appendix J). To obtain correct Reynolds stresses for the seen quantities in the original and principalaxes coordinate systems, we set

$$
w_{i}^{\prime n}=\sqrt{\left\langle w_{i}^{\prime 2}\right\rangle} \xi_{(i)}^{n} \text { and } \quad \boldsymbol{u}\left(\boldsymbol{x}^{n}\right)=\langle\boldsymbol{u}\rangle+\boldsymbol{V} \boldsymbol{w}^{\prime n} .
$$

Likewise, for the substantial derivative of the fluid-phase velocity

$$
\mathrm{d} w_{i}^{\prime n}=\sqrt{\left\langle w_{i}^{\prime 2}\right\rangle} \mathrm{d} \xi_{(i)}^{n} \quad \text { and } \quad \frac{\mathrm{D} \boldsymbol{u}}{\mathrm{D} t}=\boldsymbol{V} \frac{\mathrm{d} \boldsymbol{w}^{\prime n}}{\mathrm{~d} t}
$$

are used. In (2.21) and (2.22), brackets round indices are used to suppress Einstein summation. Note that $\langle\boldsymbol{u}\rangle, \boldsymbol{V}, \boldsymbol{W}$, and $T_{L}^{*}$ change as particles travel from grid cell to grid cell. These changes are smooth, however, since the computational grid is supposed to resolve variations of statistical quantities and particles do not travel over several grid cells during one time step. Minier et al. (2004, equation (33)) have formulated a random process for the seen fluid-phase velocity directly. Since this process is based on $k$, anisotropy in the fluid-phase turbulence is not taken into account. Moreover, consistency between the seen and fluid-phase velocity statistics is not enforced.

The time scale of changes in the seen fluid velocity, $T_{L}^{*}$, depends on the Stokes number and external forces acting on particles (Minier \& Peirano 2001). For $S t \rightarrow 0$ and in the absence of external forces, particles are passively convected by the fluid flow, i.e. $\left\langle\left|\boldsymbol{u}\left(\boldsymbol{x}^{n}\right)-\boldsymbol{v}^{n}\right|\right\rangle \rightarrow 0$, and $T_{L}^{*}$ reduces to the Lagrangian correlation time scale $T_{L}$ of fluid-phase particles. In the ballistic motion limit that applies for $S t \rightarrow \infty$ or for strong external forces acting on particles, the relative velocity $\left\langle\left|\boldsymbol{u}\left(\boldsymbol{x}^{n}\right)-\boldsymbol{v}^{n}\right|\right\rangle \gg \sqrt{2 k\left(\boldsymbol{x}^{n}\right) / 3}$ and, consequently, the correlation time scale is determined based on the frozen turbulence approximation (Pope 2000, equation (6.203)) by the Eulerian time scale $T_{E} . T_{E}$ is determined by the relative velocity between the particle and fluid phases and the flow-field correlation length scale. A model for the correlation time scale of the seen fluid velocity that attains the limits $T_{L}$ and $T_{E}$ was proposed by Minier et al. (2004, p. 2423). More recently, He et al. (2005) and Jung et al. (2008) have shown by means of DNS that away from these limits, preferential concentration effects lead to considerable changes in $T_{L}^{*}$. In this respect, Jung et al. (2008, equation (29)) have proposed the model

$$
\frac{T_{L}^{*}}{T_{L}}=0.245 \exp \left[\ln \left(\frac{S t}{C_{T 1}}\right) \frac{1}{1.3}\right]^{2}+\frac{1+(0.025 S t)^{1.5} T_{E} / T_{L}}{1+(0.025 S t)^{1.5}} .
$$

As illustrated in figure 1, the second term dominates for $S t \rightarrow 0$ and $\infty$ leading to $T_{L}^{*}=T_{L}$ and $T_{E}$, respectively. The first term in (2.23) on the other hand accounts for a prolongation of $T_{L}^{*}$ due to preferential concentration. Jung et al. (2008) have determined the parameter value $C_{T 1}=1.2$ based on their DNS results. In this work, 


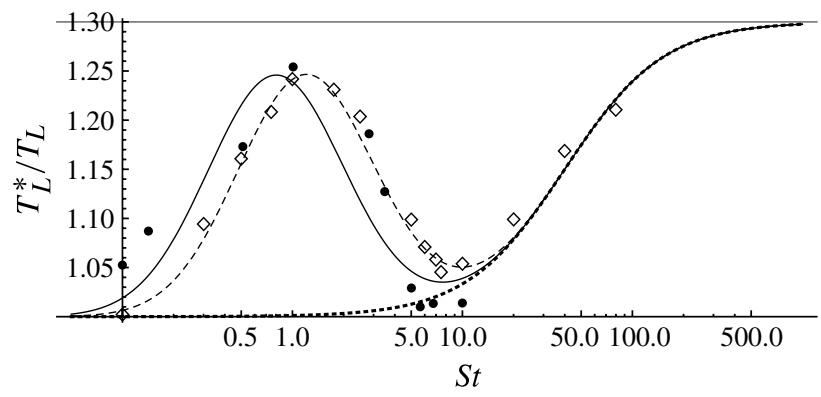

FIGURE 1. The lines show the dependence of $T_{L}^{*}$ on the Stokes number as modelled by (2.23) for $T_{E} / T_{L}=1.3$. $T_{L}^{*}(S t)$ is plotted for two different $C_{T 1}$ parameter values, i.e. $C_{T 1}=0.8$ used in this work (solid line), and $C_{T 1}=1.2$ proposed by Jung et al. (2008) based on their DNS data (dashed line). The dotted line corresponds to $T_{L}^{*}(S t)$ resulting from (2.23) with the first term removed. The symbols represent the DNS results by: $\diamond$, Jung et al. (2008) and $\bullet$, He et al. (2005).

$C_{T 1}=0.8$ is applied, which leads to good agreement of (2.23) with the DNS data of Jung et al. (2008) and He et al. (2005) as seen in figure 1.

The development of Jung et al. (2008) is based on DNS with one-way coupling and accordingly is valid for small mass loadings and does not take into account turbulence modulation effects. We expect that the prolongation of $T_{L}^{*}$ due to preferential concentration is amplified with increasing $\Phi_{m}$, since particles form clusters with increasing inertia that sweep surrounding fluid along. In this respect, we propose the following generalization of model (2.23):

$$
\frac{T_{L}^{*}}{T_{L}}=0.245 \exp \left[\ln \left(\frac{S t}{C_{T 1}}\right) \frac{1}{1.3}\right]^{2}\left(1+C_{T 2} \Phi_{m}\right)+\frac{1+(0.025 S t)^{1.5} T_{E} / T_{L}}{1+(0.025 S t)^{1.5}},
$$

where $C_{T 2}$ is a model parameter to be determined.

In a last step, closure expressions for the time scales $T_{L}$ and $T_{E}$ are presented. Based on an analysis involving the Lagrangian structure function, Pope (1994, pp. 33-34) has shown that the fluid-particle correlation time scale $T_{L}$ can be modelled as

$$
T_{L}=\frac{4}{3 C_{0}} \frac{k}{\varepsilon}
$$

with $C_{0}$ being a Reynolds-number-dependent model parameter. To determine the Eulerian time scale, we apply the model $T_{E}=\tau \equiv k / \varepsilon$. As seen in figure $1, T_{E}$ becomes important in the modelling of $T_{L}^{*}$ for large Stokes numbers. Refined models for $T_{E}$ that take the relative velocity between the fluid and particle phases into account are conceivable (Minier et al. 2004, p. 2423) but of reduced importance in the present work with $S t \ll 100$.

In summary, the Lagrangian particle-phase model consists of the particle equations of motion (2.4) and model equations (2.20)-(2.22) for the seen fluid-phase velocity with the generalized correlation-time-scale model (2.24). The latter is based on $T_{L}$ and $T_{E}$ given in the previous paragraph.

\subsection{Anisotropic turbulence dissipation model}

Poelma et al. (2007) have performed experiments with small volume and mass loadings of solid particles dispersed in water. They have demonstrated that mean 
relative motion between the phases induced by external gravity forces can lead to significant anisotropy in the fluid-phase dissipation tensor $\varepsilon_{i j}$. More specifically, the dissipation rate parallel to the mean relative velocity reduces, whereas the perpendicular components increase (Poelma et al. 2007, figure 10). However, the turbulent kinetic energy decay remains unchanged and therefore extra dissipation seems virtually absent at the low mass loadings investigated. Next, a model is presented that accounts (unlike the standard model $\varepsilon_{i j}=(2 / 3) \varepsilon \delta_{i j}$ ) for dissipation anisotropy due to the particle phase.

Poelma et al. (2007, § 5.3) have suggested using the Stokes load defined as

$$
\Phi_{S t} \equiv \Phi_{v} \frac{6}{\pi} \frac{\eta^{3}}{d^{3}} S t
$$

to relate the dissipation rate anisotropy to the underlying particle- and fluid-phase parameters. $\Phi_{S t}$, however, does not include the mean relative velocity between the phases which is causing the anisotropy. We propose the non-dimensional mass-velocity-size coefficient,

$$
\mathrm{MVS} \equiv \Phi_{m} \sqrt{\frac{|\langle\boldsymbol{u}\rangle-\langle\boldsymbol{v}\rangle|}{\sqrt{2 k / 3}}} \frac{\eta}{\mathrm{d}},
$$

and demonstrate in the Appendix that the MVS coefficient is to a good approximation linearly correlated with the dissipation rate anisotropy observed in the experiments of Poelma et al. (2007).

In a first step, a model formulation for the case where the mean relative velocity vector $\langle\boldsymbol{u}\rangle-\langle\boldsymbol{v}\rangle$ is aligned with the $x_{1}$ coordinate is outlined. For this case, we propose to model the dissipation tensor with elements $\varepsilon_{i j}$ by

$$
\tilde{\boldsymbol{E}} \equiv\left(\left[\begin{array}{ccc}
2(1-\kappa) & 0 & 0 \\
0 & \kappa-1 & 0 \\
0 & 0 & \kappa-1
\end{array}\right]+\boldsymbol{I}\right) \frac{2 \varepsilon}{3} .
$$

Here, $\boldsymbol{I}$ is the identity matrix, $\kappa \equiv 1+C_{a} \mathrm{MVS}$ and $C_{a}$ is a model constant. With increasing MVS coefficient, $\kappa$ grows and $\varepsilon_{11}$ becomes smaller while $\varepsilon_{22}$ and $\varepsilon_{33}$ increase. The trace of $\tilde{\boldsymbol{E}}$ is given by $\varepsilon_{i i}=2 \varepsilon$. This is consistent with definitions (2.16) and (2.18) of $\varepsilon_{i j}$ and $\varepsilon$, respectively, if $\tilde{\varepsilon}=\varepsilon$, which is virtually always the case (Pope 2000, bottom of p. 132). Therefore, in agreement with the findings of Poelma et al. (2007), the model (2.28) does not change the dissipation rate of $k$. Moreover, the formulation was chosen such that $\kappa=\varepsilon_{22} /\left(\varepsilon_{i i} / 3\right)$ and therefore $\kappa$ can be viewed as an anisotropy ratio.

To generalize the model (2.28) for scenarios where the coordinate system is not aligned with the mean relative velocity, the transformation $\boldsymbol{R} \equiv\left[\boldsymbol{r}_{1}, \boldsymbol{r}_{2}, \boldsymbol{r}_{3}\right]^{\mathrm{T}}$ with unity vectors

$$
\boldsymbol{r}_{1} \equiv \frac{\langle\boldsymbol{u}\rangle-\langle\boldsymbol{v}\rangle}{|\langle\boldsymbol{u}\rangle-\langle\boldsymbol{v}\rangle|}, \quad \boldsymbol{r}_{2} \equiv \frac{\boldsymbol{r}_{1} \times \boldsymbol{e}}{\left|\boldsymbol{r}_{1} \times \boldsymbol{e}\right|}, \quad \text { and } \quad \boldsymbol{r}_{3} \equiv \boldsymbol{r}_{1} \times \boldsymbol{r}_{2}
$$

is introduced. The first vector $\boldsymbol{r}_{1}$ is parallel to the relative velocity vector $\langle\boldsymbol{u}\rangle-\langle\boldsymbol{v}\rangle$ and the second vector $\boldsymbol{r}_{2}$ is normal both to $\boldsymbol{r}_{1}$ and $\boldsymbol{e}$. Vector $\boldsymbol{e}$ is any eigenvector of the Reynolds stress tensor $\boldsymbol{U}$ that is not parallel to $\boldsymbol{r}_{1}$. The transformation $\boldsymbol{R}$ is constructed 
such that the velocity vector $\boldsymbol{u}$ in the coordinate system $\left(x_{1}, x_{2}, x_{3}\right)^{\mathrm{T}}$ is mapped by

$$
\tilde{\boldsymbol{u}}=\boldsymbol{R} \boldsymbol{u}
$$

to a coordinate system $\left(\tilde{x}_{1}, \tilde{x}_{2}, \tilde{x}_{3}\right)^{\mathrm{T}}$ whose $\tilde{x}_{1}$-direction is parallel to the relative velocity vector. Conversely, $\boldsymbol{R}$ can be applied to map the model dissipation tensor $\tilde{\boldsymbol{E}}$ - whose contribution is determined in the $\tilde{\boldsymbol{x}}$ coordinate system - to the $\boldsymbol{x}$ coordinate system with

$$
\boldsymbol{E}=\boldsymbol{R}^{\mathrm{T}} \tilde{\boldsymbol{E}} \boldsymbol{R}
$$

Next, it is demonstrated that expression (2.31) is a tensor. Matrix $\tilde{\boldsymbol{E}}$ does not change if the coordinate system is changed by means of a linear transformation, say $\boldsymbol{A}$. However, vector $\boldsymbol{e}$ and the relative velocity vector change; for example, $\overline{\boldsymbol{e}}=\boldsymbol{A} \boldsymbol{e}$. Therefore, in the new coordinate system, $\boldsymbol{R}$ transforms into $\overline{\boldsymbol{R}}=\left[\boldsymbol{A} \boldsymbol{r}_{1}, \boldsymbol{A} \boldsymbol{r}_{2}, \boldsymbol{A} \boldsymbol{r}_{3}\right]^{\mathrm{T}}=$ $\left(\boldsymbol{A}\left[\boldsymbol{r}_{1}, \boldsymbol{r}_{2}, \boldsymbol{r}_{3}\right]\right)^{\mathrm{T}}=\boldsymbol{R} \boldsymbol{A}^{\mathrm{T}}$ and $\overline{\boldsymbol{R}}^{\mathrm{T}}=\boldsymbol{A} \boldsymbol{R}^{\mathrm{T}}$. Thus, we obtain

$$
\overline{\boldsymbol{E}}=\overline{\boldsymbol{R}}^{\mathrm{T}} \tilde{\boldsymbol{E}} \overline{\boldsymbol{R}}=\boldsymbol{A} \underbrace{\boldsymbol{R}^{\mathrm{T}} \tilde{\boldsymbol{E}} \boldsymbol{R}}_{=\boldsymbol{E}} \boldsymbol{A}^{\mathrm{T}},
$$

which is how a second-order tensor is supposed to transform (Pope 2000, p. 648).

In summary, the anisotropic turbulence dissipation model is based on the dissipation tensor (2.31). The two tensors appearing in (2.31) are essentially based on the mean relative velocity between the two phases and expression (2.28). The anisotropy ratio $\kappa$ that appears in (2.28) depends on the MVS coefficient (2.27).

\subsection{Model formulation for joint p.d.f. methods}

So far, a new framework to model turbulence modulation in the RANS context has been formulated. In this section, corresponding formulations for p.d.f. methods are proposed. More specifically, additions to the simplified Langevin model (SLM), which is often applied in p.d.f. methods for the simulation of turbulent flows (Pope 2000, $\S 12.3$ ), are outlined.

In p.d.f. methods, a transport equation for the joint p.d.f. of the fluid-phase velocity $\boldsymbol{u}$ is solved instead of a set of moment transport equations as in RANS methods. The p.d.f. transport equation is discussed in the textbooks by Pope (2000, $\S 12.2)$ and Fox $(2003, \S 6.5)$. A stochastic differential equation (SDE) that is equivalent to the joint velocity p.d.f. transport equation is given by

$$
\mathrm{d} u_{i}=-\frac{1}{\rho} \frac{\partial\langle p\rangle}{\partial x_{i}} \mathrm{~d} t+G_{i j}\left[u_{j}-\left\langle u_{j}\right\rangle\right] \mathrm{d} t+\sqrt{C_{0} \varepsilon} \mathrm{d} W_{i}(t),
$$

which is equation (12.110) in Pope (2000). SDE (2.33) is a model for the Lagrangian acceleration of a fluid particle, i.e. $\mathrm{d} u_{i} \approx \mathrm{D} u_{i} / \mathrm{D} t \mathrm{~d} t$ (Pope 1985, $\S \S 4$ or 4.6). $C_{0}$ is the same model constant that determines the Lagrangian time scale (2.25) and $G_{i j}$ is a model for the mean acceleration of a fluid particle. From SDE (2.33), the Reynolds stress transport equation

$$
\frac{\partial\left\langle u_{i}^{\prime} u_{j}^{\prime}\right\rangle}{\partial t}+\left\langle u_{k}\right\rangle \frac{\partial\left\langle u_{i}^{\prime} u_{j}^{\prime}\right\rangle}{\partial x_{k}}=-\frac{\partial\left\langle u_{i}^{\prime} u_{j}^{\prime} u_{k}^{\prime}\right\rangle}{\partial x_{k}}+\mathscr{P}_{i j}+G_{i k}\left\langle u_{j}^{\prime} u_{k}^{\prime}\right\rangle+G_{j k}\left\langle u_{i}^{\prime} u_{k}^{\prime}\right\rangle+C_{0} \varepsilon \delta_{i j}
$$

can be derived (compare Pope (2000, § 12.2.3)). A simple model for the specification of the tensor $G$ with elements $G_{i j}$ is the SLM which reads

$$
G_{i j}=-\left(\frac{1}{2}+\frac{3}{4} C_{0}\right) \frac{\varepsilon}{k} \delta_{i j} \text {. }
$$


To include the fluid-phase turbulence modulation effects discussed in $\S \S 2.2$ and 2.3, the SDE (2.33) is modified by including the mean Stokes drag contribution, i.e.

$$
\mathrm{d} u_{i}=-\frac{1}{\rho} \frac{\partial\langle p\rangle}{\partial x_{i}} \mathrm{~d} t+\left\langle\Phi_{m} f_{i}\right\rangle \mathrm{d} t+G_{i j}^{\prime}\left[u_{j}-\left\langle u_{j}\right\rangle\right] \mathrm{d} t+\sqrt{C_{0} \varepsilon} \mathrm{d} W_{i}(t),
$$

and by modifying $G_{i j}$ with two additions, i.e. tensor

$$
\boldsymbol{G}^{\prime}=\boldsymbol{G}+\frac{1}{2 k}\left\langle\Phi_{m} u_{i}^{\prime} f_{i}\right\rangle \boldsymbol{I}-\boldsymbol{E}^{\prime}
$$

with elements $G_{i j}^{\prime}$ is proposed as a replacement for $G_{i j}$ in (2.33). In (2.36) and (2.37), $\boldsymbol{x}$ identifies the current fluid-particle position. The last two terms in (2.37) account for turbulence attenuation and anisotropic dissipation with

$$
\boldsymbol{E}^{\prime}=\boldsymbol{R}^{\mathrm{T}} \tilde{\boldsymbol{E}}^{\prime} \boldsymbol{R} \text { and } \tilde{\boldsymbol{E}}^{\prime} \equiv\left[\begin{array}{ccc}
\frac{2(1-\kappa)}{\left\langle\tilde{u}_{1}^{\prime} \tilde{u}_{1}^{\prime}\right\rangle} & 0 & 0 \\
0 & \frac{\kappa-1}{\left\langle\tilde{u}_{2}^{\prime} \tilde{u}_{2}^{\prime}\right\rangle} & 0 \\
0 & 0 & \frac{\kappa-1}{\left\langle\tilde{u}_{3}^{\prime} \tilde{u}_{3}^{\prime}\right\rangle}
\end{array}\right] \frac{\varepsilon}{3},
$$

respectively. In $\tilde{\boldsymbol{E}}^{\prime}$, the Reynolds stresses $\left\langle\tilde{u}_{i}^{\prime} \tilde{u}_{i}^{\prime}\right\rangle$ stem from the transformed Reynolds stress tensor $\tilde{\boldsymbol{U}}=\boldsymbol{R} \boldsymbol{U} \boldsymbol{R}^{\mathrm{T}}$ (compare (2.30)). In the $\tilde{\boldsymbol{x}}$ coordinate system (defined by the transformation $\boldsymbol{R}$ based on expression (2.29)), it can be shown by insertion of expression (2.37) as a replacement for $G_{i j}$ into (2.34) that the resulting Reynolds stress dissipation tensor is equivalent to the RANS formulation (2.28). To prove that $\boldsymbol{E}^{\prime}$ is a tensor, the same arguments as outlined after (2.31) are applicable.

\section{Model validation}

In the following two sections, the turbulence modulation modelling framework that has been outlined is validated for different parameter values. In $\S 3.1$, a validation based on the DNS study of Boivin et al. (1998) is presented and in $\$ 3.2$ model comparisons with the experimental study of Poelma et al. (2007) are provided. Both cases involve particle parameters in the range $\Phi_{m} \approx 0-1$ and $S t \approx 0.1-14$.

\subsection{Attenuation of homogeneous isotropic turbulence}

\subsubsection{DNS setup}

Boivin et al. (1998) have performed DNS of forced homogeneous isotropic turbulence with different particle relaxation times $\tau_{p}$ and mass loadings $\Phi_{m}=0,0.2$, 0.5 , and 1. They have inspected the turbulent kinetic energy budget given by (2.19) and have monitored the turbulence level given by $k$ at which the forcing energy production, dissipation, and extra dissipation terms balance. Since all the different contributions to the energy budget are reported, their work provides an ideal reference to validate the Lagrangian particle-phase model (introduced in §2.2) for different particles and mass loadings.

The turbulent fluid flow in their simulations was characterized by $v=0.015 \mathrm{~m} \mathrm{~s}^{-1}$ (see Boivin et al. 1998, table 1) and the Taylor-scale Reynolds numbers

$$
\operatorname{Re}_{\lambda} \equiv \sqrt{\frac{20}{3} \frac{k^{2}}{\varepsilon v}}
$$




\begin{tabular}{lccc}
$\tau_{p}$ & \multicolumn{3}{c}{$\Phi_{m}$} \\
\cline { 2 - 4 } & 0.2 & 0.5 & 1.0 \\
0.069 & $67.19 / 5.387$ & $58.90 / 4.758$ & $45.01 / 4.028$ \\
0.251 & $66.56 / 5.046$ & $70.85 / 4.902$ & $57.58 / 4.671$ \\
0.698 & $66.95 / 4.847$ & $74.55 / 5.123$ & $67.13 / 4.721$
\end{tabular}

TABLE 1. Values of $R e_{\lambda} / \mathscr{P}_{f}$ (where $\mathscr{P}_{f}\left(\mathrm{~m}^{2} \mathrm{~s}^{-3}\right)$ is the forcing energy production rate) for different particle relaxation times $\tau_{p}$ (s) and mass loadings $\Phi_{m}$. The reported values were determined with (2.19) and (3.1) and $k, \varepsilon$, and $\mathscr{P}_{S t}$ reported in Boivin et al. (1998, figures 3,4 , and 12 ), respectively.

summarized in table 1. To maintain stationarity, the large-scale flow structures were excited by the stochastic forcing scheme outlined by Eswaran \& Pope (1988). The applied forcing energy production rates $\mathscr{P}_{f}$ are summarized in table 1. Small, heavy particles with a density ratio $\rho_{p} / \rho=2000$ (see Boivin, Simonin \& Squires 2000, table 3) and diameters $d / \eta=0.11,0.21$, and 0.35 (see Boivin et al. 1998, table 2) were used. The resulting $\tau_{p}$ values listed in table 1 were calculated with definition (1.1). To this end, the Kolmogorov length scale $\eta \equiv\left(v^{3} / \varepsilon\right)^{1 / 4}=0.0277 \mathrm{~m}$ for $\Phi_{m}=0$ was determined with the values reported in Boivin et al. (1998, table 1). Owing to the high density ratio, the pressure and viscous force term in particle equation (2.4) was not included in the DNS and the model simulations.

\subsubsection{Simulation setup}

In the simulations, the anisotropic turbulence dissipation model (introduced in $\$ 2.3$ ) drops out because on average there is no velocity difference between the two phases. Therefore, the present validation case is focused on the accurate modelling of the extra dissipation term in the $k$ budget (2.19). To determine the stationary turbulence level in the model calculations, the energy budget (2.19) was solved until a stationary state with respect to $k$ was reached. To this end, the simulator of Fuchs, Jenny \& Meyer (2010) was used as a basis. At the beginning of the simulation, $k$ was set to $7 \mathrm{~m}^{2} \mathrm{~s}^{-2}$, which is the turbulent kinetic energy reported by Boivin et al. (1998, table 1) for $\Phi_{m}=0$. For the forcing production rate $\mathscr{P}_{f}$, the values reported in table 1 were used. To calculate the dissipation rate $\varepsilon$ in (2.19), the Taylor-scale Reynolds numbers provided in table 1 were enforced: definition (3.1) leads to

$$
\varepsilon=\frac{20}{3} \frac{k^{2}}{R e_{\lambda}^{2} \nu}
$$

for the energy dissipation rate. The parameter $C_{0}$ in the model expression (2.25) for $T_{L}$ was set to 4.68 based on expression (2.25) and the values reported by Boivin et al. (1998, table 1) for the case with $\Phi_{m}=0$, i.e. $T_{L}=0.35 \mathrm{~s}, \varepsilon=5.7 \mathrm{~m}^{2} \mathrm{~s}^{-3}$, and $k=7 \mathrm{~m}^{2} \mathrm{~s}^{-2}$. The resulting $C_{0}$ is in the range of values reported by Pope (1994, pp. 54-55). In model (2.24) for the seen fluid velocity correlation time $T_{L}^{*}, C_{T 2}=23$ was found to lead to accurate predictions in the present study. To obtain reliable estimates for the extra dissipation term in (2.19), ensembles of 10000 particles were used in all simulations and the statistics were time averaged over $40 T_{L}$. Note that the particles in the model simulations represent all probable states of physical particles, but are not to be confused with the number of physical particles present in a DNS. In the Reynolds-averaged energy budget (2.19), the number of physical particles is 

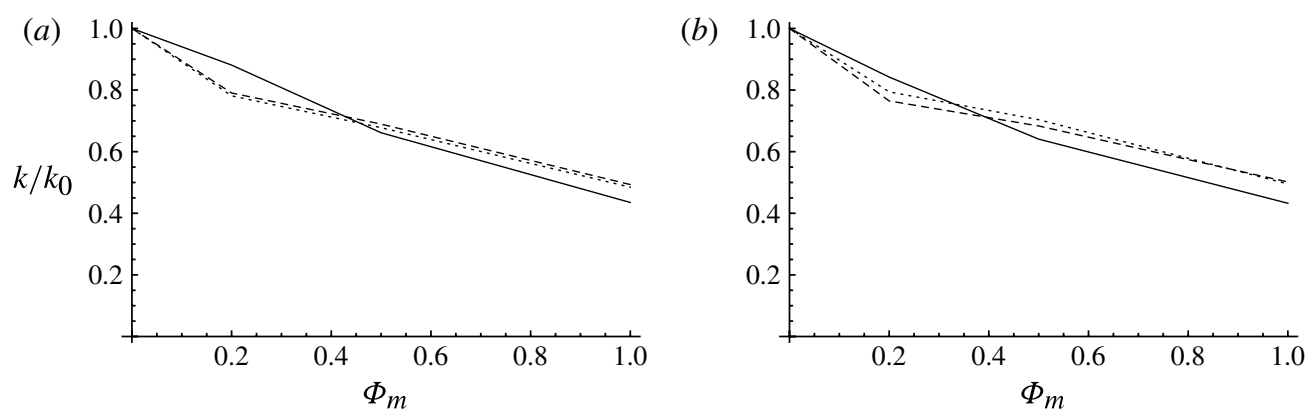

FIGURE 2. Attenuation of turbulent kinetic energy $k$ as predicted by $(a)$ the DNS of Boivin et al. (1998) and (b) the model for different mass loadings $\Phi_{m}$ and particle diameters: solid, $d / \eta=0.11, \tau_{p}=0.069 \mathrm{~s}$; dashed, $d / \eta=0.21, \tau_{p}=0.251 \mathrm{~s}$; dotted, $d / \eta=0.35, \tau_{p}=0.698 \mathrm{~s}$. The turbulent kinetic energy is normalized by $k=7 \mathrm{~m}^{2} \mathrm{~s}^{-2}$ at $\Phi_{m}=0$.
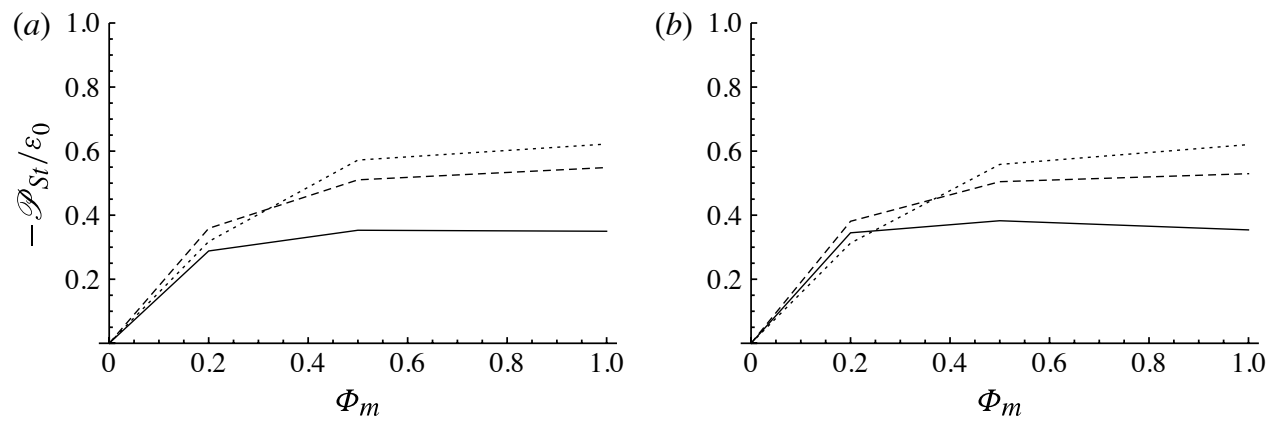

FIGURE 3. Extra dissipation $-\mathscr{P}_{S t}$ as predicted by $(a)$ the DNS of Boivin et al. (1998) and (b) the model for different mass loadings $\Phi_{m}$ and particle diameters. Line styles as in figure 2 . $-\mathscr{P}_{S t}$ is normalized by $\varepsilon=5.7 \mathrm{~m}^{2} \mathrm{~s}^{-3}$ at $\Phi_{m}=0$.

represented by the mass loading $\Phi_{m}$. At the beginning of the simulation, all $\xi_{i}^{n}$ were initialized with Gaussian random numbers with zero mean and unity variance and the $v_{i}^{n}$ were set equal to $\sqrt{2 k / 3} \xi_{i}^{n}$. For the time integration of (2.19) and (2.20), forward Euler schemes with a time step $\mathrm{d} t=0.001 \mathrm{~s}$ were used. The Wiener process increment $\mathrm{d} W(t)$ was discretized with a normal random variable with zero mean and standard deviation equal to $\sqrt{\mathrm{d} t}$ (Gardiner 2004, § 3.8.1).

\subsubsection{Results}

Based on the $\mathscr{P}_{f}$ and $R e_{\lambda}$ values taken from the DNS and the Lagrangian particlephase model outlined in $\S 2.2$, predictions for different particle relaxation times and mass loadings can be made. In figure 2, the turbulent kinetic energies that resulted from the model computations are compared with the corresponding DNS results. For the case with $\tau_{p}=0.069 \mathrm{~s}$ and $\Phi_{m}=0.2, k$ is somewhat too small but otherwise there is very good overall agreement. These observations are supported by the extra dissipation predictions provided in figure 3. For $\tau_{p}=0.069 \mathrm{~s}$ and $\Phi_{m}=0.2$, the extra dissipation is larger in the model simulations compared to the DNS. For all other particles and mass loadings, $-\mathscr{P}_{S t}$ is in very good agreement and the Lagrangian particle-phase model with the generalized correlation-time-scale model (2.24) performs 

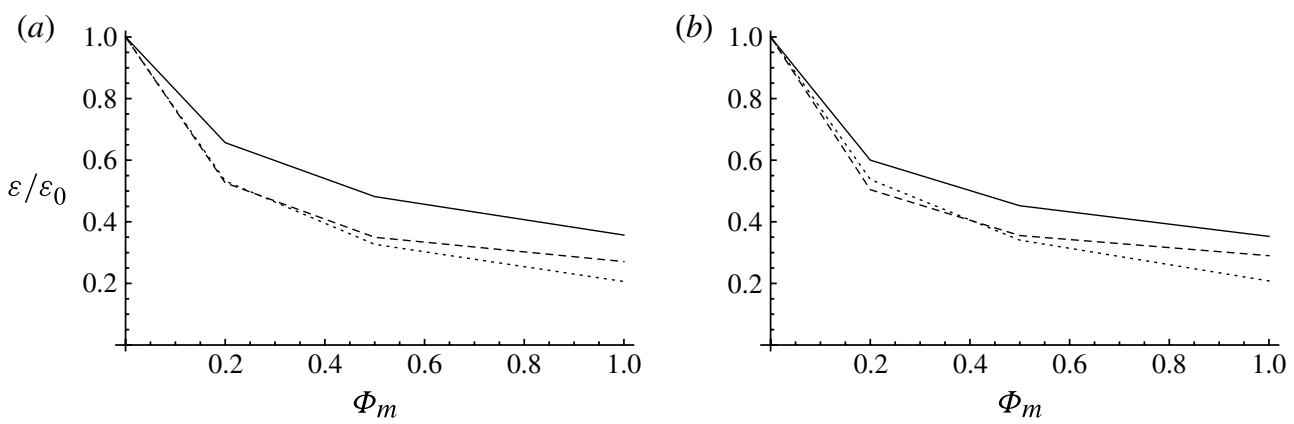

FIGURE 4. Dissipation of turbulent kinetic energy $k$ as predicted by $(a)$ the DNS of Boivin et al. (1998) and (b) the model for different mass loadings $\Phi_{m}$ and particle diameters. Line styles as in figure 2. The dissipation rate is normalized by $\varepsilon=5.7 \mathrm{~m}^{2} \mathrm{~s}^{-3}$ at $\Phi_{m}=0$.
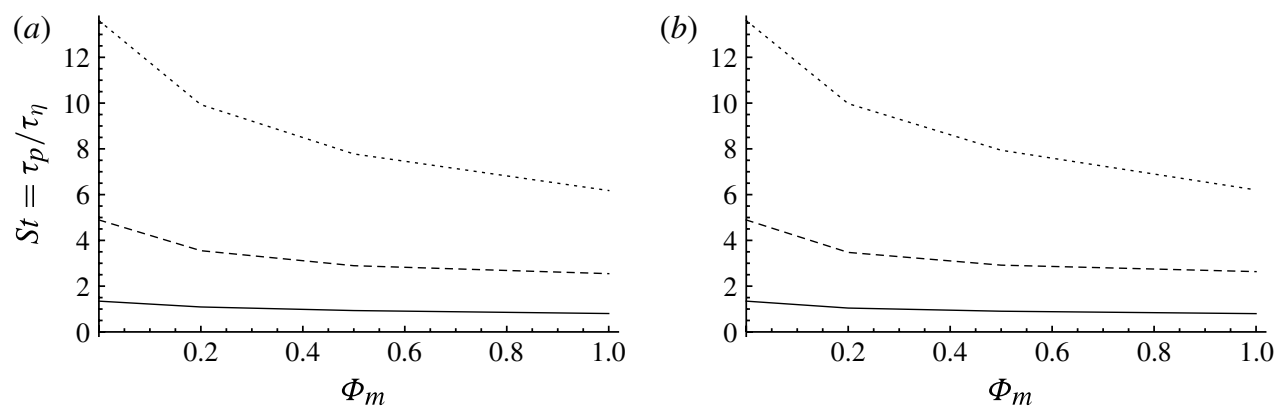

FIGURE 5. Stokes numbers as predicted by $(a)$ the DNS of Boivin et al. (1998) and $(b)$ the model for different mass loadings $\Phi_{m}$ and particle diameters. Line styles as in figure 2 .

very well. Given the fact that $k$ is accurately predicted and that $R e_{\lambda}$ is taken from the DNS, it is not surprising that the dissipation rates calculated from (3.2) are in very good agreement with the DNS results as seen in figure 4 . The Stokes numbers $S t \equiv \tau_{p} / \tau_{\eta}$ in the model simulations and the DNS with the Kolmogorov time scale $\tau_{\eta} \equiv \sqrt{\nu / \varepsilon}$ are reported in figure 5. The very good agreement of St between the model and the DNS is expected from the very good agreement observed in $\varepsilon$. The importance of the generalized correlation-time-scale model (2.24) is illustrated in figure 6. Here, simulation results with the first term in (2.24) removed are provided. Except for $\tau_{p}=0.698 \mathrm{~s}$, where $S t \approx 10$ as seen in figure 5 and consequently preferential concentration effects are small (compare figure 1), the model results are significantly different from the DNS. Therefore, it seems crucial to account for preferential concentration effects if $S t \approx 1$ as for the cases with $\tau_{p}=0.069 \mathrm{~s}$ and $\tau_{p}=0.251 \mathrm{~s}$.

\subsection{Gravitational settling of particles in decaying grid turbulence}

\subsubsection{Experimental setup}

To validate the anisotropic turbulence dissipation model presented in $\S 2.3$ and to further test the applicability of the Lagrangian particle-phase model in a spatially inhomogeneous scenario, an attempt is made to reproduce the gravity-driven flow investigated by Poelma et al. (2007). They have studied anisotropic dissipation effects 

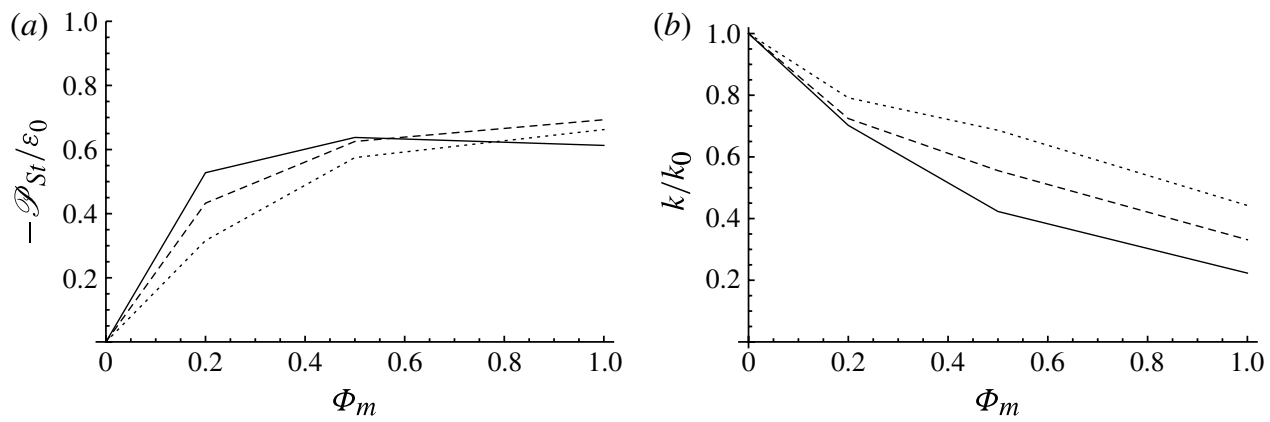

FIGURE 6. Extra dissipation $-\mathscr{P}_{S t}(a)$ and turbulent kinetic energy $k(b)$ as predicted by the model with the first term in the $T_{L}^{*}$ model (2.24) removed. Line styles as in figure $2 .-\mathscr{P}_{S t}$ and $k$ are normalized by $\varepsilon=5.7 \mathrm{~m}^{2} \mathrm{~s}^{-3}$ and $k=7 \mathrm{~m}^{2} \mathrm{~s}^{-2}$ at $\Phi_{m}=0$, respectively.

$\begin{array}{cccrccccccc}\text { No. } & \begin{array}{c}d \\ (\mu \mathrm{m})\end{array} & \begin{array}{c}\rho_{p} \\ \left(\mathrm{~kg} \mathrm{~m}^{-3}\right)\end{array} & \begin{array}{c}\tau_{p} \\ (\mathrm{~ms})\end{array} & S t & \begin{array}{c}|\langle\boldsymbol{u}\rangle-\langle\boldsymbol{v}\rangle| \\ \left(\mathrm{m} \mathrm{s}^{-1}\right)\end{array} & \begin{array}{c}\Phi_{v} \\ (\%)\end{array} & \begin{array}{c}\Phi_{m} \\ (\%)\end{array} & \begin{array}{c}\sqrt{2 k_{0} / 3} \\ \left(\mathrm{~m} \mathrm{~s}^{-1}\right)\end{array} & \begin{array}{c}\omega_{0} \\ \left(\mathrm{~s}^{-1}\right)\end{array} & \begin{array}{c}L_{D} \\ (\mathrm{~m})\end{array} \\ 1 & 153 & 3800 & 5.0 & 0.07 & 0.0246 & 0.12 & 0.44 & 0.00805 & 1.59 & 0.45 \\ 2 & 280 & 3800 & 16.6 & 0.23 & 0.0604 & 0.10 & 0.38 & 0.00901 & 1.73 & 0.5 \\ 3 & 254 & 2450 & 8.8 & 0.12 & 0.0366 & 0.26 & 0.65 & 0.01033 & 2.80 & 0.675 \\ 4 & 254 & 2450 & 8.8 & 0.12 & 0.0366 & 0.072 & 0.18 & 0.00879 & 1.89 & 0.6 \\ 5 & 280 & 3800 & 16.6 & 0.23 & 0.0604 & 0.18 & 0.67 & 0.00904 & 1.73 & 0.5\end{array}$

TABLE 2. Particle- and fluid-phase parameters from five experiments conducted by Poelma et al. (2007, tables 3 and 4). The fluid-phase boundary conditions $k_{0}$ and $\omega_{0}$ were extracted from Poelma et al. (2007, figures 16-18).

in a solid/liquid two-phase system. In their experiments, particles were settling in a channel with constant cross-section under gravity at constant mean velocities relative to a parallel, upward, uniform fluid flow. Measurements were taken in a small section in the channel centre to minimize boundary layer effects (Poelma et al. 2007, pp. 326-327). In all experiments, the mean relative settling velocity was more than 8 times smaller in magnitude than the mean upward fluid flow and, therefore, the particles were also moving upward in all cases.

The parameters of the first five experiments that were conducted by Poelma et al. (2007) are reported in table 2. Poelma et al. (2007) have provided Reynolds stress evolution data for experiments 1-6, but experiment 6 involved significant turbulence augmentation due to particle wakes. This effect is not captured by the present models and therefore experiment 6 was excluded from the present validation. The fluidphase density and kinematic viscosity were $\rho=1000 \mathrm{~kg} \mathrm{~m}^{-3}$ and $v=10^{-6} \mathrm{~m}^{2} \mathrm{~s}^{-1}$, respectively. The particles were uniformly distributed in the test section and the mean fluid flow velocity was $\langle\boldsymbol{u}\rangle=\left(0.53 \mathrm{~m} \mathrm{~s}^{-1}, 0,0\right)^{\mathrm{T}}$ in all experiments. The mean relative velocities between the particle and fluid phases, i.e. $|\langle\boldsymbol{u}\rangle-\langle\boldsymbol{v}\rangle|=\left\langle u_{1}\right\rangle-\left\langle v_{1}\right\rangle$, were constant in the flow domain (Poelma 2004, p. 136) and are reported in table 2. At the inflow boundary (or the virtual origin in the experiments (Poelma et al. 2007, figure 10)) at $x_{1}=0$, the fluid-phase Reynolds stress tensor is isotropic. The location of the turbulence generating grid, however, was approximately 35 grid spacings $M$ upstream of $x_{1}=0$. Moreover, the root-mean-square particle-phase velocity at $x_{1}=0$ 
was isotropic and equal to the fluid-phase value (Poelma et al. 2007, figure 7). The corresponding values, i.e. $\sqrt{2 k_{0} / 3}$, are listed in table 2 and were extracted from Poelma et al. (2007, figures 16-18). Also the dissipation rates at the inflow boundary, $\varepsilon_{0}$, or more precisely $\omega_{0}=\varepsilon_{0} / k_{0}$ are listed in table 2 and were estimated based on the slope of $k$ at the inflow from Poelma et al. (2007, figures 16-18).

\subsubsection{Simulation setup}

For the simulations presented in this section, the single-phase p.d.f. code of Meyer \& Jenny (2007) was enhanced by two-phase flow functionality. In a next step, the general model formulations presented in $\S 2$ are simplified for the case under consideration. Since the particle distribution is uniform and the mean flow velocities for both phases are to a good approximation constant (Poelma 2004, p. 136); (Poelma et al. 2007, §2.5), it suffices to focus on statistics of the fluctuating velocity components. From (2.4), we obtain a corresponding equation for the fluctuating particle velocity, i.e.

$$
\frac{\mathrm{d} \boldsymbol{v}^{\prime n}}{\mathrm{~d} t}=-\frac{c}{\tau_{p}}\left[\boldsymbol{v}^{n}-\boldsymbol{u}\left(\boldsymbol{x}^{n}\right)\right]+\frac{1}{\tau_{p}}\left\langle c\left[\boldsymbol{v}^{n}-\boldsymbol{u}\left(\boldsymbol{x}^{n}\right)\right]\right\rangle+\frac{\rho}{\rho_{p}}\left(\frac{\mathrm{D} \boldsymbol{u}}{\mathrm{D} t}-\frac{\partial\left\langle\boldsymbol{u}^{\prime} u_{1}^{\prime}\right\rangle}{\partial x_{1}}\right)
$$

since

$$
\left\langle\frac{\mathrm{D} u_{i}}{\mathrm{D} t}\right\rangle=\frac{\partial\left\langle u_{i}^{\prime} u_{1}^{\prime}\right\rangle}{\partial x_{1}}
$$

and with $x_{1}$ being the only direction of spatial inhomogeneity and $\langle\boldsymbol{u}\rangle$ being constant in time and space. The fluid-phase equations (2.36) and (2.37) reduce to

$$
\mathrm{d} u_{i}^{\prime}=\frac{\partial\left\langle u_{i}^{\prime} u_{1}^{\prime}\right\rangle}{\partial x_{1}} \mathrm{~d} t+G_{i j}^{\prime} u_{j}^{\prime} \mathrm{d} t+\sqrt{C_{0} \varepsilon} \mathrm{d} W_{i}(t)
$$

with

$$
\boldsymbol{G}^{\prime}=\boldsymbol{G}+\frac{\Phi_{m}}{2 k}\left\langle u_{i}^{\prime} f_{i}\right\rangle \boldsymbol{l}-\boldsymbol{E}^{\prime} \quad \text { and } \quad \boldsymbol{R}=\boldsymbol{I} .
$$

The first term on the right-hand side of (3.5) results from subtracting the mean from (2.36) or more precisely

$$
\mathrm{d} u_{i}^{\prime}=\mathrm{d} u_{i}-\left\langle\frac{\mathrm{D} u_{i}}{\mathrm{D} t}\right\rangle \mathrm{d} t=\mathrm{d} u_{i}-\frac{\partial\left\langle u_{i}^{\prime} u_{j}^{\prime}\right\rangle}{\partial x_{j}} \mathrm{~d} t
$$

This is consistent with SDE (7.59) given by Fox (2003, § 7.4.2). To determine the dissipation rate $\varepsilon$, the gamma distribution model of Jayesh \& Pope (1995) for the turbulence frequency $\langle\omega\rangle=\varepsilon / k$ was applied. Here, an instantaneous turbulence frequency $\omega$ is attributed to every fluid particle and the SDE

$$
\mathrm{d} \omega=-(\omega-\langle\omega\rangle) \frac{\mathrm{d} t}{T_{\omega}}-\langle\omega\rangle \omega S_{\omega}+\sqrt{\frac{2 \sigma^{2}\langle\omega\rangle \omega}{T_{\omega}}} \mathrm{d} W(t) \quad \text { with } T_{\omega}=\frac{1}{C_{3}\langle\omega\rangle}
$$

is solved for every particle (Pope 2000, § 12.5.3). For the flow under consideration with $\langle\boldsymbol{u}\rangle$ being constant, the source term $S_{\omega}$ simplifies to $C_{\omega 2}$ with $C_{\omega 2}, C_{3}$, and $\sigma$ being model constants. Details about the numerical time integration of the SDEs and the estimation of position-dependent statistics like $k\left(x_{1}\right)$ and $\varepsilon\left(x_{1}\right)$ (based on $\left\langle\omega\left(x_{1}\right)\right\rangle$ ) are provided in Meyer \& Jenny $(2007, \S 4.1)$. For the calculation of the Kolmogorov length scale that appears in the MVS coefficient (2.27), the definition $\eta \equiv\left(v^{3} / \varepsilon\right)^{1 / 4}$ 
with $\varepsilon\left(x_{1}\right)$ was applied. Given the small Stokes numbers, the particle- and seen fluidphase velocities at the inflow were initialized as equal, i.e. $v_{i}^{\prime n}=\sqrt{\left\langle u_{i}^{\prime 2}\right\rangle} \xi_{(i)}^{n}$. (For the flow under consideration, the Reynolds stress tensor $\boldsymbol{W}$ in (2.21) and (2.22) is equal to $\boldsymbol{U}$ and the transformation $\boldsymbol{V}=\boldsymbol{I}$.) By inspecting the simulation results, it was found that the correlation between the two velocities is high in the entire domain as expected and therefore setting $v_{i}^{\prime n}=\sqrt{\left\langle u_{i}^{\prime 2}\right\rangle} \xi_{(i)}^{n}$ at the inflow is reasonable. Since the Stokes numbers and mass loadings are small, the correlation time scale (2.24) was simplified to $T_{L}^{*}=T_{L}$. To reduce the statistical error in the results, time averaging was applied after the statistics in each simulation had reached a stationary state in time.

For the simulations of experiments 1-5, the domain sizes $L_{D}$ as listed in table 2 were used. In all simulations, the domain was discretized with 100 grid cells and $100^{2}$ computational particles were used for each phase. To initialize the fluctuating velocities of the particles of both phases at the isotropic inflow, the turbulent kinetic energies $k_{0}$ reported in table 2 were applied. The turbulence frequencies of the fluid-phase particles were initialized such that $\langle\omega\rangle=\omega_{0}$, which are also provided in table 2. Both $k_{0}$ and $\omega_{0}$ at the virtual origin were extracted from Poelma et al. (2007, figures 16-18) based on linear fits for $1 / k\left(x_{1}\right)$. Subsequently, $\omega_{0}=\varepsilon_{0} / k_{0}$ was determined based on

$$
\varepsilon=-\frac{\mathrm{d}}{\mathrm{d} t} k\left(x_{1}=\left\langle u_{1}\right\rangle t\right)=-\left\langle u_{1}\right\rangle \frac{\mathrm{d} k}{\mathrm{~d} x_{1}} .
$$

Relation (3.9) is applicable if grid turbulence is approximated as decaying homogeneous turbulence in a moving reference frame (Pope 2000, §5.4.6). To determine the time step size, a CFL number of $1 / 4$ was applied in connection with the mean velocity $\left(\Delta t \approx 2.5 \times 10^{-3} \mathrm{~s}\right)$. For the velocity $\operatorname{SDE}(3.5)$ and the gamma distribution model (3.8), standard values for the model constants were applied, i.e. $C_{0}=2.1$ (Pope 2000, p. 504), $C_{3}=1$, and $\sigma^{2}=1 / 4$ (Pope 2000, $\S 12.5 .3$ ). However, $C_{\omega 2}$ was calibrated based on the data reported by Poelma et al. (2007, table 2) for single-phase flow. It was found that $C_{\omega 2}=0.96$ leads to very good agreement as is seen in figure 7 for both the turbulent kinetic energy $k$ and turbulence frequency $\langle\omega\rangle$. Here, to normalize the downstream coordinate $x_{1}$, the grid spacing $M=7.5 \mathrm{~mm}$ reported by Poelma et al. $(2007, \S 2.1)$ was used. The anisotropy model parameter $C_{\alpha}$ was set to 28 . This value leads to very good agreement for experiment 1 and was used for all simulations.

\subsubsection{Results}

In figures 8 and 9, the anisotropic dissipation and the Lagrangian particle-phase models are validated with the experimental data of Poelma et al. (2007). A second set of simulation runs with the extra dissipation term in SDE (3.5) removed showed no significant difference with respect to the results presented in figures 8 and 9. Therefore, unlike in the DNS case studied in $\$ 3.1$ and in agreement with the conclusions of Poelma and coworkers, the extra dissipation represented by the Lagrangian particlephase model plays a minor role in their setup. On the other hand, strong anisotropic dissipation effects are visible in figures 8 and 9 and there is very good overall agreement between the experimental data and the model predictions. The Reynolds stress evolutions are predicted correctly and depending on $\operatorname{MVS}\left(x_{1}\right)$, the anisotropic dissipation model regulates the level of anisotropy. For example, a comparison of experiments 3 and 4 , where the mass loading $\Phi_{m}$ was reduced by a factor of 3.7, shows a reduction of anisotropy in experiment 4 that is captured by the model. Comparing cases 3 and 5 , where $\Phi_{m}$ is almost equal but the mean relative velocity 

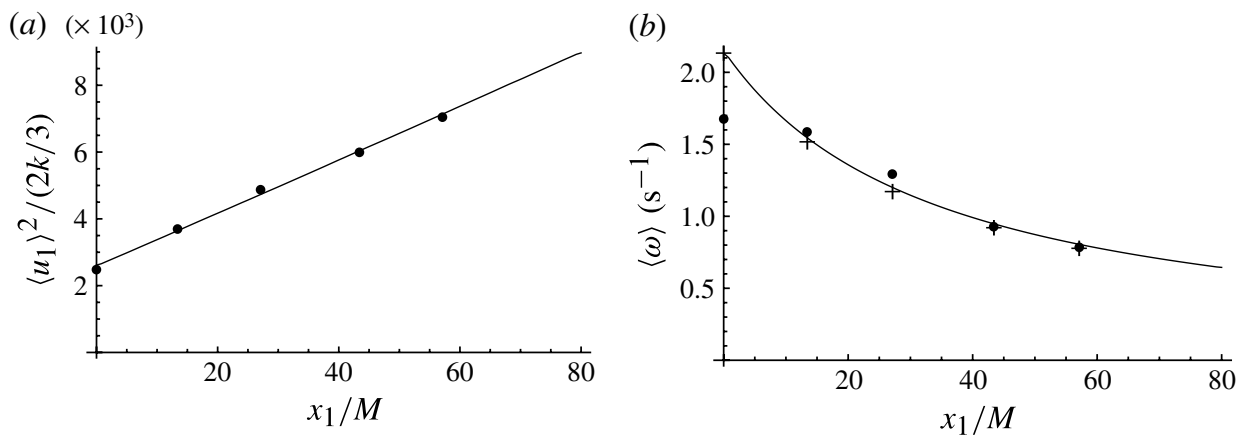

FIGURE 7. Validation of single-phase flow simulation results (lines) with experimental data of Poelma et al. (2007, table 2) (solid circles). The normalized inverse of the turbulent kinetic energy $(a)$ and the turbulence frequency $(b)$ are compared. The values reported by Poelma and coworkers for the turbulence frequency or more precisely the dissipation rate needed to be correct by a factor of $2 / 3$ (C. Poelma 2011, personal communication). The crosses in $(b)$ represent the results obtained from a linear fit to the experimental data in $(a)$ and application of (3.9).

differs by a factor of 1.7 , reveals that the mean relative velocity has a reduced effect on the anisotropy level; and similarly for experiments 1 and 2 , where the small change of $\Phi_{m}(\times 0.86)$ almost compensates for the larger change in the mean relative velocity $(\times 2.5)$. The effect of $\Phi_{m}$ is also visible in experiments 2 and 5, where the particle loading was varied. The model captures all these variations very well. It does not, however, include a mechanism for turbulence augmentation due to vortex shedding in particle wakes. In experiment 5, this effect is most probably responsible for a stabilization of the Reynolds stresses for $x_{1} / M>40$ as documented in figure $9(b)$ (Poelma et al. 2007, § 5.4). This limitation is causing the most pronounced deviations in the present validation.

\section{Conclusions}

In the present study, a modelling framework that accounts for turbulence modulation in particle- or droplet-laden flows is outlined. The framework consists essentially of two sub-models. The first component is a Lagrangian particle-phase model that mimics particle dynamics and can be used to calculate mean drag and extra dissipation effects acting on the fluid phase. The model accounts for preferential concentration effects that become important for moderate Stokes numbers. The second sub-model reproduces anisotropic dissipation effects of the Reynolds stresses that originate from a mean relative velocity between the phases. To this end, a new non-dimensional group was introduced to parametrize the anisotropic dissipation effect. Formulations of both models for RANS and joint p.d.f. methods are presented.

The performance of both models was verified in two test cases. Both cases deal with simple canonical flows, which minimizes interference with other models, e.g. turbulence models or turbulence augmentation models, and thus facilitates a thorough validation of the proposed modelling framework. The first validation case uses DNS data of stationary homogeneous isotropic turbulence as a reference. DNS data sets with particles suspended at different mass loadings and with different relaxation times were used. The model accurately quantifies the particle extra dissipation for the entire range of relaxation times and mass loadings. Accurate modelling of the seen fluid 


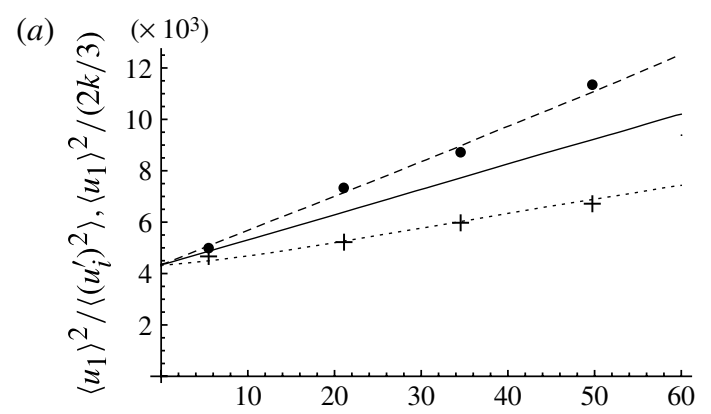

(b)

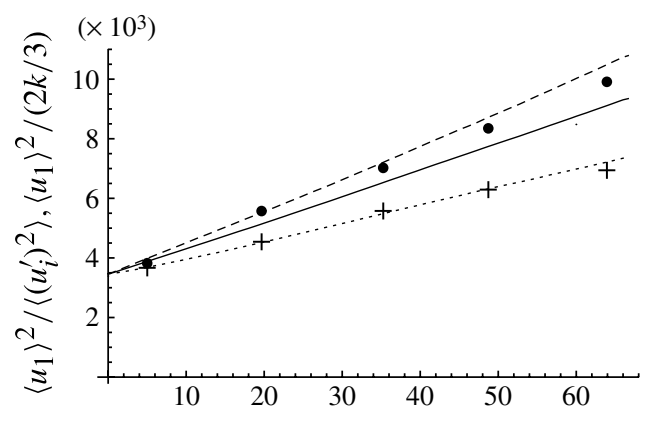

(c)

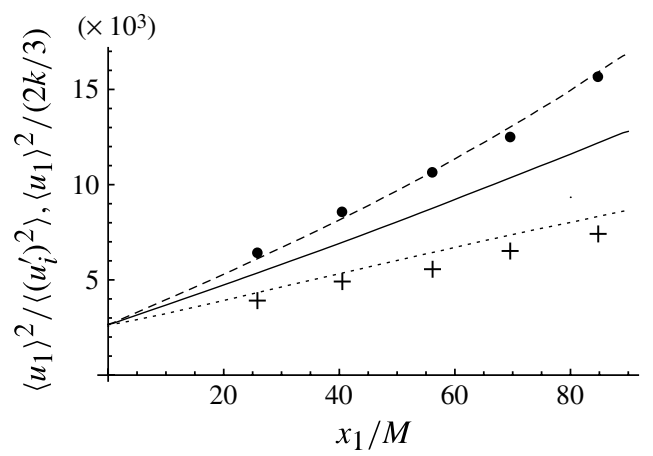

FIGURE 8. Validation of Reynolds stress evolutions resulting from two-phase flow simulations (lines) and experiments by Poelma et al. (2007, figures 16-18) (symbols). Results of $(a)$ experiment $1,(b)$ experiment 2 , and $(c)$ experiment $3 .\left\langle u_{1}\right\rangle^{2} /\left\langle u_{1}^{\prime} u_{1}^{\prime}\right\rangle$ (dotted lines and crosses), $\left\langle u_{1}\right\rangle^{2} /\left\langle u_{3}^{\prime} u_{3}^{\prime}\right\rangle$ (dashed lines and dots), and $\left\langle u_{1}\right\rangle^{2} /(2 k / 3)$ (solid lines).

velocity correlation time scale proved to be crucial for particles with short relaxation times where preferential concentration effects are important. In the second validation case, experimental data of particles settling under gravity in decaying grid turbulence were used as a reference. The resulting anisotropy in the dissipation rate tensor and the Reynolds stresses was accurately reproduced by the proposed framework for the different particle classes and mass loadings investigated.

The presented work can be generalized in several ways. For example, in applications involving flows with inhomogeneous particle distributions, the particle mass loading in (2.11) and (2.13) needs to be estimated by counting particles in grid cells of the computational domain. Moreover, a generalization of the Lagrangian particle-phase model for polydisperse particles is straightforward. In the anisotropic dissipation model, on the other hand, the particle size $d$ influences the MVS coefficient. To 

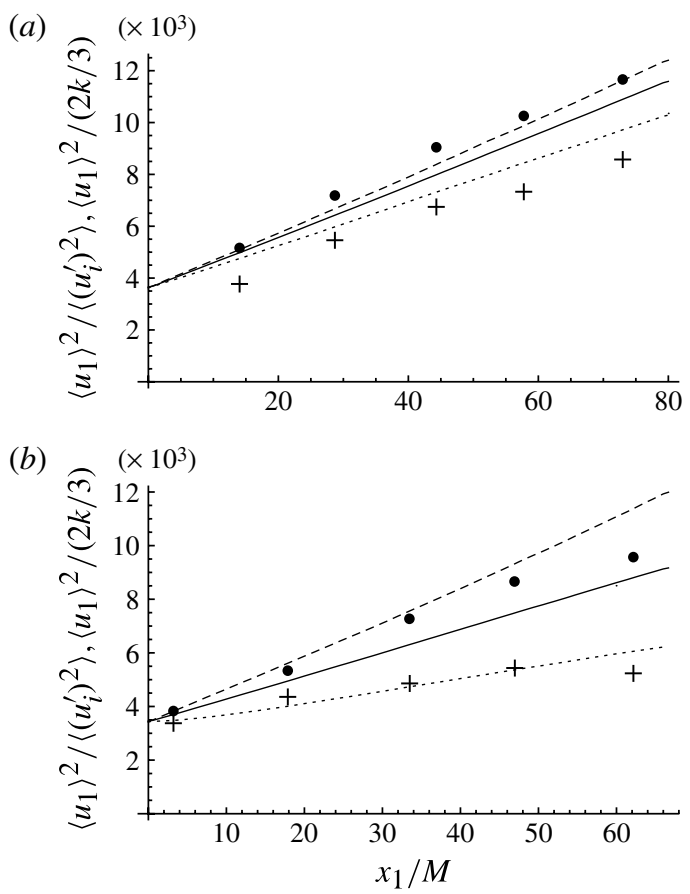

FIGURE 9. As figure 8 but for $(a)$ experiment 4 and $(b)$ experiment 5.

account for size distributions - quantified by the local size p.d.f. $f_{d}(y ; \boldsymbol{x})$ with sample space variable $y$-we suggest applying an average MVS coefficient of the form

$$
\overline{\mathrm{MVS}}=\int_{0}^{\infty} \operatorname{MVS}(d=y) f_{d}(y ; \boldsymbol{x}) \mathrm{d} y .
$$

In a Lagrangian particle-phase method, where physical particles are represented by computational particles, statistics such as $f_{d}(y ; \boldsymbol{x})$ are readily available. Finally, the proposed generalization of the correlation-time-scale model (2.24), where a linear mass loading dependence was introduced, should be verified by means of DNS.

\section{Acknowledgements}

The author thanks R. Fuchs and T. Chadha for their help during the preparation of this manuscript.

\section{Appendix. Mass-velocity-size coefficient}

Poelma et al. (2007, figure 19) have proposed the Stokes load given by definition (2.26) to parametrize particle-generated dissipation anisotropy. The mean relative velocity between the phases that causes and determines the anisotropy, however, is not represented in the Stokes load $\Phi_{S t}$. In the absence of a mean relative velocity, like in the DNS case discussed in $\S 3.1, \Phi_{S t}$ may be well above zero, but nevertheless the flow is isotropic. Here, we introduce the mass-velocity-size (MVS) 


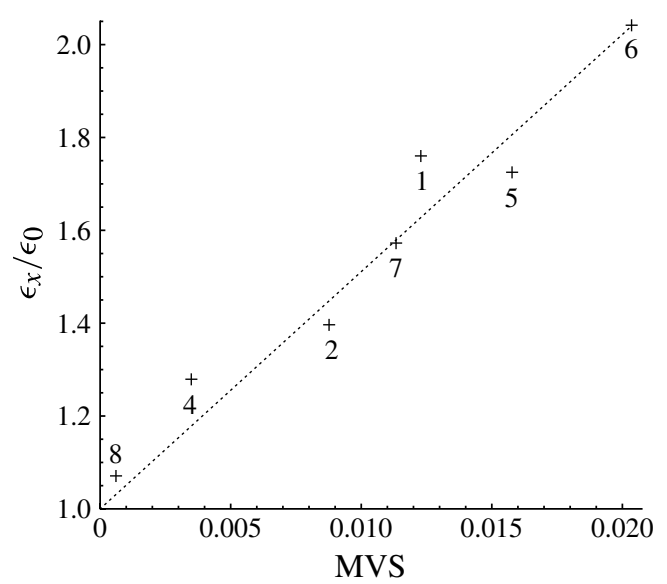

FiguRe 10. Anisotropy ratios $\epsilon_{x} / \epsilon_{0}$ (Poelma et al. 2007, p. 343) versus MVS coefficients for the experiments reported by Poelma et al. (2007, tables 3, 4 and figure 19). The labels identify each experiment and correspond with the numbers in table 2 of this work and table 4 of Poelma et al. (2007).

coefficient,

$$
\mathrm{MVS} \equiv \Phi_{m} \sqrt{\frac{|\langle\boldsymbol{u}\rangle-\langle\boldsymbol{v}\rangle|}{\sqrt{2 k / 3}}} \frac{\eta}{d},
$$

as an alternative non-dimensional parameter. It is based, like $\Phi_{S t}$, on the volume loading $\Phi_{v}$, the density ratio $\rho_{p} / \rho$, and the particle diameter $d / \eta$, but also includes the mean relative velocity $|\langle\boldsymbol{u}\rangle-\langle\boldsymbol{v}\rangle|$.

Based on the data reported by Poelma et al. (2007, tables 3, 4 and figure 19) the anisotropy ratio $\epsilon_{x} / \epsilon_{0}$ (Poelma et al. 2007, p. 343) for all experiments can be plotted as a function of the MVS coefficient. The corresponding data points for the different experiments are depicted in figure 10 and illustrate that there is a linear correlation between the MVS coefficient and $\epsilon_{x} / \epsilon_{0}$. (Experiment 3 is not included in figure 10 since it could not be identified in Poelma et al. (2007, figure 19). Experiments 7 and 8 were not included in $\$ 3.2$ because no Reynolds stress data were provided in Poelma et al. (2007) for these experiments.)

\section{REFERENCES}

BAgChI, P. \& BAlachandar, S. 2004 Response of the wake of an isolated particle to an isotropic turbulent flow. J. Fluid Mech. 518, 95-123.

Balachandar, S. \& Eaton, J. K. 2010 Turbulent dispersed multiphase flow. Annu. Rev. Fluid Mech. 42, 111-133.

Boivin, M., Simonin, O. \& Squires, K. D. 1998 Direct numerical simulation of turbulence modulation by particles in isotropic turbulence. J. Fluid Mech. 375, 235-263.

Boivin, M., Simonin, O. \& Squires, K. D. 2000 On the prediction of gas-solid flows with two-way coupling using large eddy simulation. Phys. Fluids 12 (8), 2080-2090.

Eaton, J. K. 2006 Turbulence modulation by particles. In Multiphase Flow Handbook (ed. C. T. Crowe). Taylor and Francis.

EAton, J. K. 2009 Two-way coupled turbulence simulations of gas-particle flows using point-particle tracking. Intl J. Multiphase Flow 35, 792-800. 
Eaton, J. K. \& Fessler, J. R. 1994 Preferential concentration of particles by turbulence. Intl J. Multiphase Flow 20 (1), 169-209.

ElghobASHI, S. 1993 On the two-way interaction between homogeneous turbulence and dispersed solid particles. i: turbulence modification. Phys. Fluids A 5 (7), 1790.

Elghobashi, S. \& Truesdell, G. C. 1992 Direct simulation of particle dispersion in a decaying isotropic turbulence. J. Fluid Mech. 242, 655-700.

ESWARAN, V. \& POPE, S. B. 1988 Direct numerical simulations of the turbulent mixing of a passive scalar. Phys. Fluids 31 (3), 506-520.

Fernando, H. J. S. \& ChOI, Y. J. 2007 Particle Laden Geophysical Flows: from Geophysical to Sub-Kolmogorov Scales. In Particle-Laden Flow: from Geophysical to Kolmogorov Scales (ed. B. J. Geurts, H. Clercx \& W. Uijttewaal), vol. 11, pp. 407-421. Springer.

FoX, R. O. 2003 Computational Models for Turbulent Reacting Flows. Cambridge University Press.

FuCHS, R., JENnY, P. \& MEYER, D. W. 2010 Modelling turbulence modulation in homogeneous isotropic turbulence. Tech. Rep. ETH Zurich.

Gardiner, C. W. 2004 Handbook of Stochastic Methods for Physics, Chemistry and the Natural Sciences, 3rd edn. Springer.

Geiss, S., Dreizler, A., Stojanovic, Z., Chrigui, M., Sadiki, A. \& Janicka, J. 2004 Investigation of turbulence modification in a non-reactive two-phase flow. Exp. Fluids 36 (2), 344-354.

He, Z., Liu, Z., Chen, S., Weng, L. \& Zheng, C. 2005 Particle behaviour in homogeneous isotropic turbulence. Acta Mechanica Sin. 21 (2), 112-120.

JAYESh \& POPE, S. B. 1995 Stochastic model for turbulent frequency. Tech. Rep. FDA 95-05. Cornell University.

Jung, J., YeO, K. \& LEE, C. 2008 Behavior of heavy particles in isotropic turbulence. Phys. Rev. E 77 (1), 016307.

Loth, E. 2000 Numerical approaches for motion of dispersed particles, droplets and bubbles. Prog. Energy Combust. Sci. 26, 161-223.

Meyer, D. W. \& Jenny, P. 2007 Consistent inflow and outflow boundary conditions for transported probability density function methods. J. Comput. Phys. 226 (2), 1859-1873.

Minier, J.-P. \& PeIRAnO, E. 2001 The p.d.f. approach to turbulent polydispersed two-phase flows. Phys. Rep. 352 (1-3), 1-214.

Minier, J.-P., Peirano, E. \& Chibbaro, S. 2004 P.d.f. model based on Langevin equation for polydispersed two-phase flows applied to a bluff-body gas-solid flow. Phys. Fluids 16 (7), $2419-2431$.

Poelma, C. 2004 Experiments in particle-laden turbulence. Doctoral thesis, Technische Universiteit Delft.

Poelma, C., Westerweel, J. \& Ooms, G. 2007 Particle-fluid interactions in grid-generated turbulence. J. Fluid Mech. 589, 315-351.

Pope, S. B. 1985 P.d.f. methods for turbulent reactive flows. Prog. Energy Combust. Sci. 11 (2), 119-192.

Pope, S. B. 1994 Lagrangian pdf methods for turbulent flows. Annu. Rev. Fluid Mech. 26, 23-63.

Pope, S. B. 2000 Turbulent Flows. Cambridge University Press.

Press, W. H. 2001 Numerical Recipes in Fortran 77 the Art of Scientific Computing, 2nd edn.. Cambridge University Press.

Rogers, C. B. \& EATON, J. K. 1991 The effect of small particles on fluid turbulence in a flat-plate, turbulent boundary layer in air. Phys. Fluids A 3 (5), 928-937.

Shaw, R. A. 2003 Particle-turbulence interactions in atmospheric clouds. Annu. Rev. Fluid Mech. 35, $183-227$.

SquiRes, K. D. \& EATON, J. K. 1990 Particle response and turbulence modification in isotropic turbulence. Phys. Fluids A 2 (7), 1191-1203.

Squires, K. D. \& EATON, J. K. 1991a Measurements of particle dispersion obtained from direct numerical simulations of isotropic turbulence. J. Fluid Mech. 226, 1-35.

Squires, K. D. \& Eaton, J. K. $1991 b$ Preferential concentration of particles by turbulence. Phys. Fluids A 3 (5), 1169-1178.

Sundaram, S. \& Collins, L. R. 1997 Collision statistics in an isotropic particle-laden turbulent suspension. Part 1. Direct numerical simulations. J. Fluid Mech. 335, 75-109. 